\title{
Robust Monetary Policy with Misspecified Models: Does Model Uncertainty Always Call for Attenuated Policy?
}

\author{
Robert J. Tetlow and Peter von zur Muehlen*
}

\begin{abstract}
This paper explores Knightian model uncertainty as a possible explanation of the considerable difference between estimated interest rate rules and optimal feedback descriptions of monetary policy. We focus on two types of uncertainty: (i) unstructured model uncertainty reflected in additive shock error processes that result from omitted-variable misspecifications, and (ii) structured model uncertainty, where one or more parameters are identified as the source of misspecification. For an estimated forward-looking model of the U.S. economy, we find that rules that are robust against uncertainty, the nature of which is unspecifiable, or against one-time parametric shifts, are more aggressive than the optimal linear quadratic rule. However, policies designed to protect the economy against the worst-case consequences of misspecified dynamics are less aggressive and turn out to be good approximations of the estimated rule. A possible drawback of such policies is that the losses incurred from protecting against worst-case scenarios are concentrated among the same business cycle frequencies that normally occupy the attention of policymakers.
\end{abstract}

JEL Classification: C6, E5

Keywords: model uncertainty, robust control, monetary policy, Stackelberg games.

${ }^{*}$ Federal Reserve Board, Washington, DC 20551. Tetlow: rtetlow@frb.gov or by mail to stop 61; von zur Muehlen: pmuehlen@frb.gov or by mail to stop 76. Forthcoming, the Journal of Economic Dynamics \& Control. We thank David Reifschneider and three anonymous referees for useful guidance and especially Alexei Onatski for generously providing two prototypical Matlab programs and subsequent help in understanding the methodology. Thanks are also due to Frederico S. Finan for his usual, excellent research assistance. All remaining errors are ours. Any opinions expressed herein do not necessarily reflect those of the staff or members of the Board of Governors. 


\section{Introduction}

Recent articles have uncovered a puzzle in monetary policy: Interest-rate reaction functions derived from solving optimization problems call for much more aggressive responsiveness of policy instruments to output and inflation than do rules estimated with US data. ${ }^{1}$ What explains the observed lack of aggressiveness-the attenuation-of policy?

Three distinct arguments have been advanced to explain the observed reluctance to act aggressively. The first is that it is simply a matter of taste: Policy is slow and adjusts smoothly in response to shocks because central bankers prefer it that way, either as an inherent taste or as a device to avoid public scrutiny and criticism (see, e.g., Drazen (2000), Chapter 10). The second argues that partial adjustment in interest rates aids policy by exploiting private agents' expectations of future short-term rates to move long-term interest rates in a way that is conducive to monetary control (see, e.g., Goodfriend (1991), Woodford (1999), Tetlow and vonzur Muehlen (2000)).

The third contention is that attenuated policy is the optimal response of policymakers facing uncertainty in model parameters, in the nature of stochastic disturbances, in the data themselves given statistical revisions, and in the measurement of latent state variables such as potential output, the NAIRU, and the steady-state real interest rate. Blinder (1998), Estrella and Mishkin (1998), Orphanides (1998), Rudebusch (1998), Sack (1998a), Smets (1999), Orphanides et.al. (2000), Sack and Wieland (2000), Wieland (1998), and Tetlow (2000) all support this general argument, following the line of research that began with Brainard (1967). ${ }^{2}$. The present paper is concerned with this third explanation for policy attentuation. There is no unanamity on this third line of argument, however. Chow (1975) and Craine (1979) demonstrated long ago that uncertainty can lead to the opposite result of more aggressive policy than in the certainty equivalence case — or what we might dub as anti-attenuation. Söderström (1999a) provides an empirical example of such a case. Moreover, possible deficiencies in the Brainard-style story are hinted at in the range of uncertainties required in papers by Sack (1998a) and Rudebusch (1998) to come even close to explaining observed policy behavior. ${ }^{3}$ Lastly, time-variation in uncertainty can, in some circumstances, lead to anti-attentuation of policy as shown by Mercado and Kendrick (1999).

The concept of model uncertainty underlying the papers cited above is Bayesian in nature:

\footnotetext{
${ }^{1}$ The list of papers includes Rudebusch (1998), Sack (1998b), Söderström (1999b), Tetlow, von zur Muehlen, and Finan (1999) and Tetlow and von zur Muehlen (2000).

${ }^{2}$ Other important early references include Aoki (1967), Johansen (1973), Johansen (1978), and Craine (1979)

${ }^{3}$ Rudebusch (1998) finds that data uncertainty has only a slight attenuating effect on optimal policy. Similarly, Onatski and Stock (2000) find that data uncertainty has a minimal effect on reactions formed when monetary policy is robust in the sense of the term described below.
} 
A researcher faces a well-defined range of possibilities for the true economy over which he or she must formulate a probability distribution function (see Easley and Kiefer (1988)). All of the usual laws from probability theory can be brought to bear on such questions. These are problems of risk, and risks can be priced. More realistically, however, central bankers see themselves as facing far more profound uncertainties. They seem to view the world as so complex and time varying that the assignment of probability distributions to parameters or models is impossible. Perhaps for this reason, no central bank is currently committed to a policy rule (other than an exchange rate peg). In acknowledgment of this view, this paper arises from the conception of uncertainty, in the sense of Knight, wherein probability distributions for parameters or models cannot be articulated.

We consider two approaches to model uncertainty that differ in the nature of the specification errors envisioned and in the robustness criterion applied to the problem. One approach treats errors as manifested in arbitrarily serially correlated shock processes, in addition to the model's normal stochastic disturbances. This formulation, called unstructured model uncertainty, follows in the tradition of Caravani and Papavassilopoulos (1990) and Hansen, Sargent and Tallarini (1999), among others. A second approach puts specific structure on misspecification errors in selected parameters in a model. It is possible, for example, to analyze the effect on policy of the worst possible one-time shift in one or more parameter. Alternatively, misspecification in model lag structures could be examined. Such unmodeled dynamics, will affect robust policy. The seminal economics paper in this area of structured model uncertainty is Onatski and Stock (2000).

The inability to characterize risk in probability terms compels the monetary authority to protect losses against worst-case outcomes, to play a mental game against nature, as it were. In the case of unstructured uncertainty, the solution to the game is an $\mathcal{H}^{\infty}$ problem or, in a related special case, a problem that minimizes absolute deviations of targets. In the case of structured uncertainty, the monetary authority ends up choosing a reaction function that minimizes the chance of model instability. In both cases, the authority adopts a bounded "worst-case" strategy, planning against nature's "conspiring" to produce the most disadvantageous parameterization of the true model.

With the exception of Hansen and Sargent (1999b), Kasa (2000), and Giannoni (2000), robust decision theory has been applied solely to backward looking models. Hansen and Sargent (1999b) and Kasa (2000) derive policies under the assumption of unstructured uncertainty, while Giannoni (2000) solves a problem with structured uncertainty, wherein policies are derived subject to uncertainty bounds on selected parameters of the model. In this paper, we break new ground in that we consider a number of cases of unstructured as well as structured 
uncertainty, doing so for an estimated forward-looking model, and with a particular real-world policy issue in mind. Also, unlike Hansen and Sargent (1999b) and Kasa (2000), but like Giannoni (2000) and Onatski and Stock (2000), we derive robust simple policy rules, similar in form to the well-known Taylor (1993) rule. Our analysis differs from Giannoni (2000) in that it is less parametric, relies on numerical techniques, and is amenable to treatment of larger models and covers unstructured as well as structured uncertainty.

The rest of this paper unfolds as follows. In section 2, we introduce structured and unstructured perturbations as a way of modeling specification errors to a reference model considered to be the authority's best approximation to true but unknown model. We define a number of Stackelberg games that differ according to the central bank's assessment of the bounds on uncertainty and its loss function. To analyze the specific questions at hand, in the third section, we estimate a small forward-looking macro model with Keynesian features. The model is a form of contracting model, in the spirit of Taylor (1980) and Calvo (1983), and is broadly similar to that of Fuhrer and Moore (1995a). Section 4 provides our results leaving Section 5 to sum up and conclude.

To presage the results, although we are able to produce a robust rule that is nearly identical to the estimated rule, it is not clear how much of the issue this resolves. Robustness, per se, cannot explain attenuated policy. As others have found, when policy is robust against a combination of shock and misspecification errors, monetary policy becomes even more reactive than the linear quadratic optimal rule. Indeed, we observe a seeming inverse relationship between reactiveness and the degree of structure imposed on uncertainty. At one extreme, unstructured uncertainty justifies the most reactive set of rules. At the other extreme, heavily attenuated policies are generated in cases with a lot of structure on model uncertainty. In particular, if the monetary authority chooses a policy that is robust only to misspecification of the lag structure of the model, the optimal interest rate reaction rule becomes very similar to the estimated rule. When the only criterion is robustness to misspecifications of the lagged output coefficients in the aggregate demand equation, the robust rule and the estimated rule are practically identical. It is tempting to infer from this result that Federal Reserve policy in the last twenty years has been influenced by a special concern about ill-understood future effects of current actions. However, ours is not sufficient evidence to establish that the US monetary policy authority has or has not in fact been a robust decision maker. 


\section{Model Uncertainty in the Sense of Knight}

Few observers of the U.S. macroeconomic scene in 1995 would have forecast the happy coincidence of very strong output growth coupled with low inflation that was observed over the second half of the decade. Such forecast errors tend to bring out the humble side of model builders and forecasters alike and reinforce the lesson that considerable uncertainty surrounds our understanding of the real world, a fact that should be taken account of in planning. In the presence of such pervasive ambiguity, the notion of Knightian uncertainty has an obvious appeal. Whatever the circumstances, it is easy to imagine that the best guess of the true model a policymaker can bring to the issue of monetary control is flawed in a serious but unspecifiable way. We consider an approach, founded on recent developments in robust decision theory, in which the authority contemplates approximation errors of unspecified nature but of a size contained within a bounded neighborhood of its reference model. As will become apparent shortly, this approach has the advantage of fitting into a linear quadratic framework, but without its usual association with certainty equivalence. The absence of a probability distribution for possible misspecifications leads, in turn, to a desire to minimize worst-case outcomes. Previous research has shown that this can be usefully formulated as a two-person game played, in this instance, between the monetary authority and a malevolent nature. ${ }^{4}$

It is easy to conceptualize how model misspecification can be represented as a game. Imagine a monetary authority attempting to control a misspecified model. As it does, the misspecification will manifest itself, out of sample, as residuals whose time-series pattern differs from those derived by estimating the model. The nature of these ex post residuals will depend on the nature of the misspecification, the bounds on its size, and the specifics of the feedback rule employed by the authority. However, the uncertainty involved-uncertainty in the sense of Knight - is not amenable to standard optimization techniques, reliant as they are on the means and variances of state variables. It is reasonable for a policymaker that is unable to distinguish the likelihood of good and bad events to seek protection from the worst outcomes. This lends a non-cooperative gaming aspect to the problem, with the authority planning to protect itself against the possibility that nature will throw the worst possible set of ex post shocks allowed by the bounds of the problem.

Different assumptions about uncertainty bounds alter the nature of each game in ways that

\footnotetext{
${ }^{4}$ The policy environment used in this paper is similar to one used in von zur Muehlen (1982), which examined several two-person Stackelberg games with Knightian uncertainty modeled as uniform distributions over the stochastic processes of the parameters. Early treatments of control as two-person games include Sworder (1964) and Ferguson (1967). More recent papers include Glover and Doyle (1988), Caravani and Papavassilopoulos (1990), Caravani (1995), and Basar and Bernhard (1991), Hansen et al. (1999), Hansen and Sargent(1996,1998,1999a).
} 
will be explored below. In addition, the precise formulation of each game will be determined by the amount of structure placed on approximation errors assumed to arise from misspecification. In this regard, we first assume that specification errors, viewed as perturbations to the reference model, are unstructured in the sense of being completely reflected in the additive stochastic error process driving the reference model. This uncertainty about the model is parameterized by a bound on extreme rather than average values of the loss function, which rise as unspecifiable uncertainty increases, as we will show. ${ }^{5}$ With these restrictions, the decision maker is compelled to act cautiously by assuring a minimum level of performance under the worst possible conditions. Whether or not such caution leads to less or more intensified feedback, is a question we will answer in the context of our empirical model. ${ }^{6}$

A second set of exercises, also within the framework of Knightian uncertainty, examines robust decisions under model uncertainty alone. There, we shall distinguish between uncertainty in parameters and uncertainty in dynamics. Parameter uncertainty is a fairly clear concept, except that with Knightian uncertainty we make no distributional assumptions. In addition, we shall distinguish between one-time shifts and time varying approximation errors that may be either linear or nonlinear. Dynamic misspecification, including omitted or unmodeled dynamics, is common and implies that error processes are not white noise. For such cases, we are able to use a recently developed technique, called $\mu$ analysis, to determine interest-rate rules that are robust to worst-case misspecifications of the lag structure in a model. A drawback of the solutions for robust control rules under structured uncertainty is that computational methods for obtaining unique minima of risk functions are generally not available. However, it is possible to generate robust rules resulting in output with finite norms that guarantee robust stability but not necessarily robust performance of losses. ${ }^{7}$

\subsection{A generic linear rational expectations model}

Let $x_{t}$ denote an $n \times 1$ vector of endogenous variables in the model. A number, $n_{1}$, of these variables is assumed to be expectational (nonpredetermined, forward looking), and the remainder, $n_{2}=n-n_{1}$, are predetermined (backward looking). The economy is assumed to evolve

\footnotetext{
${ }^{5}$ The literature on robust control has its genesis in the seminal paper by Zames (1981). The first systematic treatment of robust control in terms of explicit state feedback rules, based on $\mathcal{H}^{\infty}$ norms on a system's transfer functions, is by Doyle, Glover, Khargonekar and Francis (1989).

${ }^{6}$ Another way of addressing this issue is to construct, by experimentation, rules that are robust to a variety of models. An fine example of this is Levin, Wieland and Williams (1999) which tests interest-rate rules for their performance in four structural models. While these results are quite instructive, they are limited in generality by the small set of models considered. The premise of Knightian uncertainty is that such specific knowledge is lacking.

${ }^{7}$ The relevant methodology is described in Zhou, Doyle and Glover (1996) and Dahleh and Diaz-Bobillo (1995).
} 
according to the following law of motion,

$$
x_{t+1}=A x_{t}+B u_{t}+v_{t+1},
$$

where $u_{t}$ is a $k$-dimensional vector of policy instruments, and $v_{t+1}$ is a vector of random shocks, the properties of which we detail below. ${ }^{8}$ Throughout, we assume that the authority uses only one instrument and commits to the stationary rule, $u_{t}=K x_{t}$, where $K$ is an $1 \times n$ vector of parameters to be chosen. ${ }^{9}$ Let $T_{t}$ denote a target vector, for example inflation, output, and possibly the policy maker's control variable. $T$ is thus represented as the mapping,

$$
T_{t}=M_{x} x_{t}+M_{u} u_{t}
$$

For $u_{t}=K x_{t}$, the target is, $T_{t}=M x_{t}$, where $M=M_{x}+K M_{u}$ is $m \times n$. As is usually done in this literature, the periodic loss function is a quadratic form involving the target vector, $T_{t}$ : $L_{t}=T_{t}^{\prime} Q T_{t}$, where $Q$ is an $m \times m$ (diagonal) weighting matrix of fixed scalars assigned by the authority. Next, it is convenient to define the output vector,

$$
z_{t}=Q^{\frac{1}{2}} T_{t}=H x_{t}
$$

where $H=Q^{\frac{1}{2}} M$ is an $m \times n$ matrix. With this, the periodic loss function becomes,

$$
L_{t}=z_{t}^{\prime} z_{t}=x_{t}^{\prime} H^{\prime} H x_{t}
$$

The authority's objective function is the discounted sum of periodic losses,

$$
V_{0}=\sum_{t=0}^{\infty} \beta^{t} L_{t}=\sum_{t=0}^{\infty} \beta^{t} z_{t}^{\prime} z_{t},
$$

where $0<\beta \leq 1$ is a time discount factor. ${ }^{10}$ Note that, contrary to the usual practice, we do not express future losses in stochastic terms, given the definition of uncertainty as Knightian.

Given a stabilizing vector, $K$, the unique saddlepath, with exactly $n_{1}$ roots within the unit

\footnotetext{
${ }^{8}$ The vector of residuals, $v_{t+1}$ may contain zeros. Equation (1) may be thought of the solved companion form of a structural model, in which isues, such as singularities have been resolved by appropriate solution techniques.

${ }^{9}$ The feedback rule may be a "synthesized" optimal or robust control rule or it may be a restricted (simple) optimal or robust feedback rule, when some elements of $K$ are restricted to be zero.

${ }^{10}$ In the calculations, we set $\beta=1$, which we can do without loss of generality, provided we are willing to assume the existence of a commitment technology. Values less than one can be trivially accommodated by well known transformations.
} 
circle, and $n_{2}$ outside, implies the restricted reduced-form ${ }^{11}$

$$
x_{t+1}=\prod x_{t}+C v_{t+1},
$$

where $\Pi$ and $C$ depend nonlinearly on the parameters in the policy rule as well as on the structural parameters of the model. ${ }^{12}$ This non-linear dependence of the reduced-form parameters on structural and policy parameters implies that specification errors in the structural (reference) model are also reflected in the parameters of the reduced form. As is common practice, we express model uncertainty by augmenting the transition matrix - in our case, the reduced form of the reference model — with a matrix of perturbations, $\Delta_{\Pi}$, representing misspecification errors,

$$
x_{t+1}=\left(\Pi+\Delta^{\Pi}\right) x_{t}+C v_{t+1} .
$$

In the next few pages we shall tackle two cases: (1) unstructured model uncertainty, represented by combined model and shock uncertainty, in which we do not distinguish between $\Delta^{\Pi} x_{t}$ and $C v_{t}$, and (2) structured model uncertainty, in which uncertainty is solely associated with the model's parameters.

\subsection{Unstructured uncertainty}

We treat the general case of unstructured model uncertainty first, combining errors arising from independent disturbances with those that arise from misspecification. If misspecification errors are due to omitted variables, the implied additive shocks will be heteroskedastic, with additional dependence on the decision rule. Further, as noted by Hansen and Sargent (1998), by feeding back on endogenous variables, misspecified shock processes capture misspecified endogenous dynamics. If these errors in specification manifest themselves through the same dynamics as the model's additive shocks - as they would in the case of omitted variables - they may be reflected in size and autocorrelation of the residuals, possibly at frequencies that may damage the policy maker's stabilization goals. Under the assumption that the distribution of shocks is unknown, the authority chooses elements in $K$ to prevent or minimize worst outcomes to the performance metric.

Setting $w_{t+1}=\Delta^{\Pi} x_{t}+C v_{t+1}$, and combining (7) and (3), the system may be compactly

\footnotetext{
${ }^{11}$ A solution may be obtained using any of a variety of techniques for solving linear dynamic perfect foresight models. We use the Anderson-Moore algorithm, which computes saddle-point solutions using the QR decomposition to obviate problems with possible non-singularities. See Anderson and Moore (1985) and Anderson (2000).

${ }^{12}$ Note that in every instance, the computations properly account for this dependence as well as the dependence on the feedback parameters, $K$.
} 
represented by,

$$
\left[\begin{array}{l}
x_{t+1} \\
z_{t}
\end{array}\right]=\left[\begin{array}{ll}
\Pi & I \\
H & 0
\end{array}\right]\left[\begin{array}{l}
x_{t} \\
w_{t+1}
\end{array}\right]
$$

This describes a state-space system with output, $z_{t}$, and input, $w_{t+1}$, where, as noted earlier, $w_{t+1}$ should be viewed as the nature's control variable. With $w_{t+1}$ as the instrument of one of our players, we henceforth treat it not as a stochastic process but as a deterministic sequence of bounded approximation errors.

We now introduce the mapping from shocks, $w_{t}$, to the target vector, $z_{t}$, called the transfer function, $G$. It is obtained by solving (8) for $z_{t}$ as a function of $w_{t}$ :

$$
z_{t}=H(I-\Pi L)^{-1} w_{t} \equiv G w_{t}
$$

We are interested in the size of $G$, because it measures how disturbances, including perturbations to the model, affect target performance. However $G$ is measured, i.e., whatever norm we adopt, smaller values are always to be preferred to larger ones.

As first proposed by Basar and Bernhard (1991), the problem of the monetary authority facing Knightian uncertainty may be cast as a two-person game with a Nash equilibrium: nature choosing the sequence $\left\{w_{t+1}\right\}$, taking the authority's feedback rule as given, and the authority choosing the vector, $K$, which feeds back on the state variables. Using the Hansen and Sargent (1999a) formulation, the decision maker chooses $K$ to minimize welfare losses, and malevolent nature chooses $w_{t}$ to maximize welfare losses:

$$
\min _{K} \max _{w_{t}} \sum_{t=0}^{\infty} z_{t}^{\prime} z_{t}
$$

subject to (8) and

$$
\begin{aligned}
\sum_{t=0}^{\infty} w_{t}^{\prime} w_{t} & \leq \eta^{2}+w_{0}^{\prime} w_{0} \\
x_{0} & =w_{0} .
\end{aligned}
$$

The above formulation is a very general and powerful representation of a class of games. In the special case where $\eta=0$, the policy maker is solely concerned with additive shock disturbances. In the more general case where $\eta>0$, the game is determined by the initial value of the shock process, $w_{0}$, which, represents the opponent's commitment to its hostile strategy.

Several results as well as the computations in section 4 require use of the frequency domain. 
Accordingly, we introduce the following one-sided Fourier transform,

$$
X(\xi) \equiv \sum_{t=0}^{\infty} x_{t} \xi^{t}
$$

where $\xi=e^{i \omega}$, and $\omega$ is a point in the frequency range $-\pi, \pi$. Applying this transformation to (8) and (9), yields the following reformulation of the output vector, $z_{t}$, and the transfer function, $\mathcal{G}$, analogous to the transfer function, $G$, defined in the time domain,

$$
\begin{aligned}
Z(\xi) & =\mathcal{G}(\xi) W(\xi) \\
\mathcal{G}(\xi) & =H(I-\xi \Pi)^{-1}
\end{aligned}
$$

Applying Parseval's equality, the game in (10) is equivalently expressed as the finding of a pair, $[K, \mathcal{W}]$, that solves

$$
\begin{aligned}
\inf _{K} \sup _{\mathcal{W}} \sum_{t=0}^{\infty} z_{t}^{\prime} z_{t} & =\inf _{K} \sup _{\mathcal{W}} \frac{1}{2 \pi} \int_{-\pi}^{\pi} W(\xi)^{\prime} \mathcal{G}(\xi)^{\prime} \mathcal{G}(\xi) W(\xi) d \omega \\
\int_{-\pi}^{\pi} W(\xi)^{\prime} W(\xi) d \omega & \leq \eta^{2}+w_{0}^{\prime} w_{0}
\end{aligned}
$$

subject to (8). Notice that the integral is defined on the unit disk.

This formulation allows us to describe several possible games, depending on particular assumptions made about the form of the loss function and the bounds placed on Knightian uncertainty. The authority may, for various reasons, be interested in minimizing a square measure of loss, such as a quadratic form, or it may want to minimize the largest absolute deviation of its losses. In the absense of distributional assumptions, the authority must also decide how large nature's shocks can be. Typical assumptions are that shocks are square-summable or that their largest absolute value is less than some finite number. Such assumptions are typically expressed as norms. In our case, we shall be mainly interested in two such norms, denoted $\ell_{2}$ and $\ell_{\infty}$, respectively, ${ }^{13}$

\footnotetext{
${ }^{13}$ Let $l_{p}^{n}(Z)$ be the space of all vector-valued real sequences on integers of dimension $n$, where $x=$ $(\cdots, x(-1), x(0), x(1), \cdots)$ with $x(k) \in \Re^{n}$, such that

$$
\|x\|_{p}=\left(\sum_{k=-\infty}^{\infty} \sum_{j=1}^{n}\left|x_{j}(k)\right|^{p}\right)^{1 / p}<\infty .
$$

If $p=1,\|x\|_{1}$ belongs in $\ell_{1}$ and is the sum of absolute values. For $p=2$, the norm, $\|x\|_{2}$ belongs in $\ell_{2}$, and its square is the amount of "energy" in a signal, which, in statistics or economics, is akin to a covariance or quadratic loss. For $p=\infty,\|x\|_{\infty}$ belongs in $\ell_{\infty}$ and is the maximum "magnitude" or "amplitude" a signal can attain over all time.
} 
1. $\ell_{2}(X)=\left(\sum_{0}^{\infty}|X(j)|^{2}\right)^{1 / 2}$ : square-root of the sum of squares of $\mathrm{X}$; when $X=z$, this is the linear quadratic regulator (LQR) loss function in (10). The constraint shown in (11), given $\eta \neq 0$, is an $\ell_{2}$ norm, where $X=w$.

2. $\ell_{\infty}(X)=\sup _{j}|X(j)|$ : the largest absolute size of $X$. For $X=w$, it measures the maximum amplitude of disturbances.

We now present three versions of the preceding game, each distinguished from the other by an assumption made about $\eta$ and the manner in which nature is assumed to have "committed" to a strategy, $\mathcal{W}$, as manifested by its choice (or lack thereof) of the initial shock, $w_{0}$. Given nature's commitment to a strategy, $\mathcal{W}$, the authority can solve the opponent's maximization problem to eliminate $\mathcal{W}$ and reduce the problem to one involving an indirect loss function. In the language of linear operator theory, the resulting loss function, expressed as a norm on $G$, is said to be "induced". ${ }^{14}$ Of course, the central bank does not know nature's strategy other than that it is bounded in some sense. We discuss three versions of the game in (10)-(11), each determined by particular assumptions concerning the form of the loss function and the bounds placed upon Knightian uncertainty. As is shown next, by specifying which of the above norms applies to the loss function and which to the shock process, the authority determines the kind of one-player, "induced" loss function it seeks to minimize. An important feature of that indirect loss function is that is independent of the opponent's strategy, $\left\{w_{t+1}\right\}$.

Table 3 anticipates the indirect loss functions we will derive in the next three sections. The top row specifies the norm assumed to bound uncertainty, and the leftmost column shows the assumed norm for the loss function. The cells in the table display the indirect loss functions that result when one combines assumptions from the top row with loss functions at the left. The $\ell_{1}$ norm is defined in footnote 13 and in section 2.2.3.

\section{Table 1: Loss Functions Induced by Type of Unstructured Model Uncertainty}

\begin{tabular}{|c|c|c|c|}
\hline & \multicolumn{3}{|c|}{ Model Uncertainty: $\eta>0 \quad$ Certainty Equivalence: $\eta=0$} \\
\hline Underlying Loss & $\ell_{2}$ & $\ell_{\infty}$ & $\ell_{2}$ \\
\hline "Quadratic Loss: $\ell_{2}$ & $\overline{\overline{\mathcal{H}^{\infty}}}$ & - & $\overline{\overline{\mathcal{H}^{2}}}$ \\
\hline Maximum Loss: $\ell_{\infty}$ & - & $\ell_{1}$ & - \\
\hline
\end{tabular}

\footnotetext{
${ }^{14}$ Let $\mathrm{G}\left(G=\left(g_{i j}\right) \in \Re^{m \times n}\right)$ be a transfer function from $w$ to $z: z=G w$, then the $\ell_{p}$ induced norm of $G$ is an operator from $\left(\Re^{n},\left.|\cdot|\right|_{p}\right)$ to $\left(\Re^{m}\right)$,

$$
\|G\|_{p-\text { induced }}=\sup _{w \neq 0} \frac{\|G w\|_{p}}{\|w\|_{p}},
$$

which is the amount of amplification the operator $G$ exerts on the space, $Z$. Further, $G$ is a bounded operator norm from $W$ to $Z$, if its induced norm is finite, $\|G\|_{p}<\infty$.
} 


\subsection{1 $\ell_{2}$ loss without model uncertainty: the linear quadratic regulator (LQR)}

Assume that model uncertainty is not an issue, that is, let $\eta=0$ and allow $W(0)=w_{0}$ to be arbitrary. In this case, (11) is satisfied only if $w_{t}=0$ for all $t>0$, so that $W(\xi)=w_{0}$, a constant for all $\xi$. Equivalently, the shocks, $w$, have no spectral density, so that $W(\xi)^{\prime} W(\xi)=$ $I$. The game (12) reduces to the linear-quadratic problem with certainty equivalence,

$$
\begin{aligned}
\inf _{K} \sup _{\mathcal{W}} \sum_{t=0}^{\infty} z_{t}^{\prime} z_{t} & \equiv \inf _{K} \sup _{\mathcal{W}}\|z\|_{2}^{2}=\inf _{K} \sup _{\mathcal{W}} w_{0}^{\prime}\left[\frac{1}{2 \pi} \int_{-\pi}^{\pi} G(\xi)^{\prime} G(\xi) d \omega\right] w_{0} \\
& =\inf _{K} w_{0}^{\prime}\|G\|_{2}^{2} w_{0} .
\end{aligned}
$$

Minimization of the loss function is therefore equivalent to minimization of the norm $\|G\|_{2}$ in $\mathcal{H}^{2}$, (the Hardy space of square-summable analytic functions on the unit disk), where

$$
\|G\|_{2}=\frac{1}{2 \pi} \int_{-\pi}^{\pi} \operatorname{trace}\left[\mathcal{G}^{\prime}(\xi) \mathcal{G}(\xi)\right] d \omega
$$

is a function of the authority's decision rule, $K$, but not of $w$. Each point on a plot of $\operatorname{trace}\left[\mathcal{G}(\xi)^{\prime} \mathcal{G}(\xi)\right]$ represents the contribution of the shock process at frequency point $\omega$ to the total loss. Notice that $\|G\|_{2}$ is related to a generalized variance, defined by integrating over spectral frequencies with equal weighting across frequencies. As we shall show below, the robust authority does not assign equal weights to all frequencies; rather, it assigns larger weights to frequencies to which the economy is most susceptible to damage from well chosen shocks, in accordance with its strategy to avoid worst-case outcomes. Since all policies, robust or not, imply a variance measure of loss, different policies can be compared by plotting $\operatorname{trace}\left[\mathcal{G}(\xi)^{\prime} \mathcal{G}(\xi)\right]$, measuring their relative strengths and vulnerabilities at various frequencies.

\subsection{2 $\ell_{2}$ loss with $\ell_{2}$ bounded model uncertainty: the $\mathcal{H}^{\infty}$ problem}

As noted before, the LQR, with its implication of certainty equivalence, may distort policy if risk is a real concern. If the authority believes risk to be a significant feature of the environment it faces, a different approach is required. In terms of the game (12), model uncertainty is equivalent to letting $\eta>0$ be arbitrary. For the present case, also assume that $W(0)=w_{0}=0$. The initial setting, $w_{0}$ therefore disappears from the constraint, making the problem the same as if nature made no commitment to an initial $w_{0}$, at all. ${ }^{15}$ As before, the authority's nominal loss function is assumed to be quadratic. In Hansen and Sargent (1999a) and Zhou et al. (1996)

\footnotetext{
${ }^{15}$ Absence of commitment to $w_{0}$ is a hallmark of $\mathcal{H}^{\infty}$ control. As shown in subsection 2.2.4, games that are intermediate to $\mathcal{H}^{2}$ and $\mathcal{H}^{\infty}$, assume that nature does commit to some $w_{0} \neq 0$.
} 
it is shown that for (12), this problem implies a single-agent minimization problem in $\mathcal{H}^{\infty}$,

$$
\begin{aligned}
\inf _{K} \sup _{\mathcal{W}} \frac{1}{2 \pi} \int_{-\pi}^{\pi} W(\xi)^{\prime} \mathcal{G}(\xi)^{\prime} \mathcal{G}(\xi) W(\xi) d \omega & \leq \inf _{K} \sup _{\mathcal{W}} \sup _{\omega \in[-\pi, \pi]} \bar{\sigma}^{2} \frac{1}{2 \pi} \int_{-\pi}^{\pi} W(\xi)^{\prime} W(\xi) d \omega \\
& \leq \inf _{K} \sup _{\mathcal{W}} \bar{\sigma}^{2} \eta^{2}=\inf _{K}\|G\|_{\infty}^{2} \eta^{2}
\end{aligned}
$$

where $\bar{\sigma}$ denotes the singular value of $\mathcal{G}$, defined as the square root of the largest eigenvalue of $\mathcal{G}^{\prime} \mathcal{G}{ }^{16}$ The idea behind this approach is to spread the consequences of unknown serial correlations across frequencies by designing a rule that works well over a range of values of $W(\xi)^{\prime} W(\xi)$, taking the view that $\|w\|_{2}$ is the worst that nature can do. The saddlepoint solution of (14) is equivalent to the infinum of the $\mathcal{H}^{\infty}$ norm, $\|G\|_{\infty}$,

$$
\inf _{K}\|G(K)\|_{\infty}=\inf _{K} \sup _{\mathcal{W}} \frac{\|G(K)\|_{2}}{\|w\|_{2}} \equiv \bar{\theta}
$$

Denoting the minimizing feedback of $K$ by $\hat{K}, \bar{\theta}$ satisfies the inequality,

$$
\|G(\hat{K})\|_{2} \leq \bar{\theta}\|w\|_{2}, \text { for all } w \in \ell_{2}
$$

demonstrating that robust policy can limit the ratio of the two norms. Confining this ratio to acceptable levels is called disturbance attenuation in the engineering control literature. While an increase in $\bar{\theta}$ always implies an increase in the level of uncertainty, Hansen and Sargent (1999a) identify declining values of $\theta$ with rising levels of preference for robustness. Conversely, as $\theta \rightarrow \infty$, the preceding criterion converges on the standard LQR policy.

\subsection{3 $\ell_{\infty}$ loss with $\ell_{\infty}$ bounded model uncertainty: the MAD criterion}

Instead of minimizing deviations from target paths against shocks with square-summable bounds, the policy maker is now assumed to avoid worst-case scenarios by minimizing the maximum amplitude of target deviations, $\|z\|_{\infty}$, against the largest possible shock satisfying $\|w\|_{\infty}<\eta^{2}$. This assumption represents policies of an authority that feels especially susceptible to unfortunate shocks. This combination of loss function and size of uncertainty induces an $\ell_{1}$ indirect loss on $G$, defined as the weighted sum of absolute deviations of targets from their desired

\footnotetext{
${ }^{16} \mathrm{~A}$ referee has pointed out the dependence of $w_{t+1}$ on the feedback, $K$, as a potential problem in the analysis of $\mathcal{H}^{\infty}$ problems. Since nature may choose a $w_{t+1}$ in response to $K$ that is huge in comparison to other shocks, even if $G(K)$ amplifies this shock as little as possible, the damage can be very large. Framed in terms of the game, the variance restriction on the shocks depends on $K$, so that $\sum w^{\prime}(K) w(K)<\eta^{2}+w_{0}^{\prime} w_{0}$. Note, however, the assumption that $\eta$ is arbitrary means that the implied robust policy will guarantee adequate performance against the worst that nature can do, including reacting adversely to $K$. In any case, this feature is not confined to the forward-looking model analyzed here; the nature of the game solution makes it generic to all dynamic models.
} 
levels, $\|z\|_{1} \cdot{ }^{17}$ Formally, the minimum absolute deviation (MAD) problem solves,

$$
\begin{aligned}
& \min _{K} \sup _{w}\|G w\|_{\infty} \\
& \text { s.t }\|w\|_{\infty} \leq \eta^{2},
\end{aligned}
$$

which is equivalent to

$$
\min _{K} \sup _{w:\|w\|_{\infty} \leq \eta^{2}} \frac{\|G w\|_{\infty}}{\|w\|_{\infty}}=\min _{K}\|G\|_{1}=\min _{K} \max _{1 \leq i \leq m} \sum_{i=1}^{n}\left|G_{i j}\right| .
$$

\subsubsection{Between $\mathcal{H}^{2}$ and $\mathcal{H}^{\infty}$ : Minimum Entropy}

A digression that is useful for casting robust control in language that is familiar to economists, is the case of minimum entropy. Observe that in the $\mathcal{H}^{2}$ control problem, misspecification becomes irrelevant when $\eta=0$ and $w_{0}$ is allowed to be arbitrary. By contrast, in the $\mathcal{H}^{\infty}$ robust control problem, $\eta>0$ allows for model misspecification, while $w_{0}$ is assumed to be zero, or, equivalently, free. An intermediate case commits nature to specify an initial condition, $w_{0} \neq 0$, leading to the Lagrangian multiplier game,

$$
\inf _{K} \sup _{w:\|w\|_{2} \leq \eta^{2}+w_{0}^{\prime} w_{0}}\|z\|_{2}
$$

An interesting result due to Whittle (1990) is that the preceding game reduces to a singleplayer control problem with the authority minimizing entropy or, equivalently, a risk-sensitive function closely related to risk aversion in utility theory.

$$
\inf _{K}\left[\frac{1}{2 \pi} \int_{-\pi}^{\pi} \log \operatorname{det}\left[\left(\mathcal{G}(\xi)^{\prime} \mathcal{G}(\xi)-\theta I\right)\right] d \omega\right]=\inf _{K}-\frac{2}{\alpha} \log E\left(e^{-\frac{\alpha}{2}\|G w\|_{2}}\right),
$$

which is defined only for $\theta>\bar{\theta}$, where $\theta$ is the Lagrangian multiplier in (17), and $\bar{\theta}$ is the smallest positive scalar for which the integrand is negative semidefinite. ${ }^{18}$ The relationship between the entropy criterion and the $\mathcal{H}^{\infty}$ game is that $\bar{\theta}$ is the infinum of the $\mathcal{H}^{\infty}$ norm in

\footnotetext{
${ }^{17}$ In estimation theory, the $\ell_{1}$-norm estimator is known as the least absolute deviation estimator (LAD), proposed by Powell (1981).

${ }^{18}$ Representing model uncertainty as a game may seem like a stretch to some readers since it gives to what is normally an exogenous process the fiction of strategic non-cooperative behavior. There is, however, room to modulate this behavior by adjusting preferences for robustness to fit the problem in a specific way. In the game (17), it is the multiplier to the constraint which becomes the instrument for disciplining the perceived behavior of nature, earning it the interpretation as (the inverse of) a measure of preference for robustness. Accordingly, nature, seeking to maximize losses, is penalized if $w^{\prime} w \geq \eta^{2}+w_{0}^{\prime} w_{0}$. The added criterion of robustness is equivalent to the introducton of a pessimistic attitude, $w^{\prime} w$, the importance of which is governed by $\theta$ : small values of $\theta$ imply a $w$ that can be large, while large values of $\theta$ make nature's threat less important.
} 
(15). The parameter, $\alpha=-1 / \theta$, has an interesting interpretation as a Knightian risk sensitivity parameter. The relationship between risk sensitivity and robustness is further illuminated by expanding the last term above in powers of $\alpha<0$,

$$
\log E\left(e^{-\frac{\alpha}{2}\|G w\|_{2}}\right) \approx E_{K}\left(\|G w\|_{2}\right)+\frac{\alpha}{4} \operatorname{var}_{K}\left(\|G w\|_{2}\right)+O\left(\alpha^{2}\right)
$$

where $E_{K}$ generates a mathematical expectation, and $\operatorname{var}_{K}$ is the variance operator. As $\alpha$ approaches $-1 / \bar{\theta}$ from above, (becoming larger in absolute value), dislike of increasing values of $\|G w\|_{2}$ rises. Conversely, as $\alpha$ approaches 0 , the problem increasingly reduces to the traditional linear quadratic control problem, as the variance term disappears. Of course, a decrease in $\bar{\alpha}$ of the $\mathcal{H}^{\infty}$ bound itself represents a decrease in actual model uncertainty.

Finally, Hansen and Sargent (1995) have shown that (18) can be solved using the recursion,

$$
V_{t}=z_{t}^{\prime} z_{t}-\frac{2}{\alpha} \log E_{u} e^{-\frac{1}{2} \alpha V_{t+1}}
$$

which demonstrates that preference for robustness is like a discount factor, suggesting that with $\alpha \neq 0$, the authority has an incentive to forestall future consequences of current model uncertainty by acting aggressively.

\subsection{Structured model uncertainty}

\subsubsection{Defining the game}

We now turn to structured model uncertainty, where uncertainties are assigned to parameters of the model instead of being consigned to additive shock processes. For convenience we repeat equation (7) but show the lag operator, $L$, as an explicit argument of $\Pi$ to emphasize the dynamic nature of the model,

$$
x_{t+1}=\left(\Pi(L)+\Delta^{\Pi}\right) x_{t}+C v_{t+1} .
$$

Uncertainty about parameters or about unmodeled dynamics is formally treated as perturbations, $\Delta^{\Pi} \in \Delta$, where $\Delta$ is a perturbation block spanning all approximation errors. These can include one-time jumps in individual parameters, misspecifications in contemporaneous channels from policy to the state variables, and omission of critical lag structures affecting the dynamic behavior of the economy. Analytically, the structured perturbations, $\Delta^{\Pi}$, are operators defined independently of the state vector, $x_{t}$.

If the authority believes approximation errors to lie within some small neighborhood, then 
individual elements, $\Delta_{i j}^{\Pi}$, corresponding to the elements of $\Pi(L)$, will have small norms less than $r \delta_{i j}$, where $r$ is the radius of allowable perturbations, and $\delta_{i j}$ is a scaling constant assigned to the $i j$-th parameter in $\Pi(L) .{ }^{19}$ In this case the perturbation block may be defined as the diagonal matrix, $\boldsymbol{\Delta}=\operatorname{diag}\left\{\Delta_{i j}^{\Pi} / \delta_{i j}\right\}$. The admissible set of perturbations is formally denoted $D_{r}=\left\{\boldsymbol{\Delta}:\left\|\boldsymbol{\Delta}_{\mathrm{ij}}^{\Pi} / \delta_{\mathrm{ij}}\right\|<\mathbf{r}\right\}$, where $r \leq \bar{r}<\infty$. Accordingly, the set, $D_{r}$, is widened as the radius of allowable perturbations is increased. A game similar to (12) is the Stackelberg game,

$$
\min _{K} \sup _{\Delta^{\Pi} \in D_{r}}\|z\|_{2}^{2}
$$

subject to (7), where $K$ is the vector of parameters chosen by the authority, and $\Delta^{\Pi}$ is a diagonal matrix of the perturbations (controlled by nature). ${ }^{20}$ Naturally, the authority is interested in protecting itself against the widest set of perturbations, but no policy may exist that accomplishes this task. Conversely, as one narrows the range of permissible misspecifications, the menu of possible rules that achieve stability widens and may become unmanageable. As a result, the authority may seek a unique rule, that, at least for benchmark purposes, guarantees stability of the economy for the maximum range of misspecifications of parameters or lag structures.

\subsubsection{A framework for analyzing structured perturbations}

To characterize the perturbation block, $\Delta$, we may consider time-varying versus time-invariant perturbations and linear versus nonlinear perturbations. Among linear perturbations, those with the greatest amount of imposed structure are time-invariant scalar (LTI-scalar) perturbations, representing such events as one-time shifts in parameters and structural breaks. If the authority is concerned with misspecification in the lag structure of the model, then the case of infinite moving average (LTI-MA) perturbations might be the most appropriate to consider, since they involve perturbations with long memory. An authority that fears dynamic misspecification to be in the form of LTI lag polynomials seeks out policies that protect against the worst possible lag misspecifications. For LTI perturbations, the literature has developed techniques in the frequency domain, which help conserve on the number of parameters. The last in the

\footnotetext{
${ }^{19}$ In the exercises, the $\delta_{i j}$ are functions of the standard errors of estimates of the model.

${ }^{20}$ The perturbation operator, $\Delta$, is defined independently of the state, $x_{t}$. As a consequence, we analyze robust system stability, in contrast with the procedures for unstructured uncertainty, which yield policies that assure robust performance. For a backward looking model, Onatski and Stock (2000) are able to solve for performance robustness in a special case where the two criteria coincide. Onatski (1999) derives optimal minimax rules for parametric as well as lag-structure uncertainty in a backward looking model. In the present model, given forwardlooking agents and the need to work with the reduced form representation of the model, this is not possible.
} 
group of linear perturbations are time-varying (LTV) perturbations. Within the class of nonlinear perturbations, we are able to consider both nonlinear time-invariant (NTI), and nonlinear time-varying (NTV) perturbations, where the latter allow the widest latitude and greatest nonparametric generality of model uncertainty. The last three types of perturbations to the model (LTV, NTI, and NTV) can be treated as one, and we shall refer to them collectively as NTV, because, as it turns out, the stability conditions for each are identical.

Turning to the task of specifying the perturbation block, denote the space of all causal perturbations by $\ell_{\Delta}$. As before, $\Delta$, denotes the class of allowable perturbations, i.e., those that carry with them the structure information of the perturbations. In our case, $\Delta$ is the set of all diagonal perturbations of the form,

$$
\Delta=\left\{\operatorname{diag}\left(\Delta_{i j}^{\Pi} / \delta_{i j}\right) \mid \Delta_{i j}^{\Pi} \in \ell_{\Delta}\right\}
$$

where the $\Delta_{i j}^{\Pi}$, which can be any of the types of perturbations discussed before, are assumed to be $\ell_{p}$-stable, and $p=2$ or $\infty$. The subset of $\Delta$ containing elements with $\ell_{p}$ norm smaller than than $r$ is denoted $\mathbf{B}_{\Delta, p}$, so that

$$
\mathbf{B}_{\Delta, p}=\left\{\Delta^{\Pi} \in \Delta \mid\left\|\Delta^{\Pi}\right\|_{\ell_{p}-i n d}<r\right\}
$$

For reasons that will become clear presently, we represent the perturbed model as an interconnected system of equations linking the state vector, $x_{t}$, and a vector of perturbations. Let us suppose that a subset of the elements in $\Pi$ are misspecified, as represented by bounded perturbations, and suppose this involves $k$ elements of the state vector, $x_{t}$. Accordingly, let $\Gamma$ and $\mathcal{U}$ be the appropriately dimensioned selector matrices, filled with zeros and ones, that pick the correctelements of $\Pi$ to be perturbed and elements of $x_{t}$ that become involved, respectively. The perturbed and true model is,

$$
x_{t+1}=\left(\Pi+\Gamma \Delta^{\Pi} \mathcal{U}\right) x_{t}+C v_{t+1}
$$

By defining an augmented output vector, $p_{t}=\mathcal{U} x_{t}$ and a corresponding input vector, $h_{t}=$ $\Delta^{\Pi} x_{t}$, the preceding equation is equivalent to the augmented feedback loop,

$$
\begin{aligned}
{\left[\begin{array}{c}
x_{t+1} \\
p_{t}
\end{array}\right] } & =\left[\begin{array}{lll}
\Pi & C & \Gamma \\
\mathcal{U} & 0 & 0
\end{array}\right]\left[\begin{array}{c}
x_{t} \\
v_{t+1} \\
h_{t}
\end{array}\right], \\
h_{t} & =\Delta^{\Pi} p_{t}
\end{aligned}
$$


which is in a form amenable to the techniques to be used below.

Once again, we want to analyze the size of a transfer function, although this time around, the transfer function does not involve the target vector, per se. We solve (19) for $x_{t}$ and $p_{t}$ as a mapping from $v_{t}$ and $h_{t}$. This yields the transfer matrix, $G$, written in partitioned form,

$$
\begin{aligned}
{\left[\begin{array}{l}
x_{t} \\
p_{t}
\end{array}\right] } & =\left[\begin{array}{l}
I_{n} \\
\mathcal{U}
\end{array}\right](I-\Pi L)^{-1}\left[\begin{array}{ll}
C & \Gamma
\end{array}\right]\left[\begin{array}{l}
v_{t} \\
h_{t}
\end{array}\right], \\
& \equiv\left[\begin{array}{ll}
G_{11} & G_{12} \\
G_{21} & G_{22}
\end{array}\right]\left[\begin{array}{l}
v_{t} \\
h_{t}
\end{array}\right] .
\end{aligned}
$$

Notice that the interconnection between $h_{t}$ and $p_{t}$ is represented by two channels: feedforward $p_{t}=G_{22} h_{t}$ and feedback $h_{t}=\Delta^{\Pi} p_{t}$. Why is $G_{22}$ interesting? For an answer, appeal is made to the "small gain theorem" (see Dahleh-Diaz-Bobillo (1995) and Zhou, Doyle, and Glover (1996)), which states that for all $\Delta^{\Pi} \in \Re \mathcal{H}_{p},\left\|G_{22}\right\|_{p}<1 / r$ if and only if $\Delta^{\Pi} \leq r$. In words, if the policy rule, $K$, stabilizes the nominal model (7), then the augmented model (19)-(20) is stable if and only if the feedback interconnection between $h_{t}$ and $p_{t}$ in (21) is stable. As a consequence, only the stability of $G_{22}$ need be examined for any desired norm to assure stability of the full model, under the same criterion. For $p=2$ and $p=\infty$, the mathematics for evaluating stability under structured perturbations involves the structured norm,

$$
S N_{\Delta, p}\left(G_{22}\right)=\frac{1}{\inf _{\Delta^{\Pi}[}\left[\left|\Delta^{\Pi} \|_{\ell_{p}-\text { induced }}\right|\left(I-G_{22} \Delta^{\Pi}\right)^{-1} \text { is not } \ell_{p}-\text { stable }\right]},
$$

such that if $\left(I-G_{22} \Delta^{\Pi}\right)^{-1}$ is $\ell_{p}$-stable for every $\Delta^{\Pi} \in D_{r}$, then $S N_{\Delta, p}\left(G_{22}\right)=0$. Importantly, the maximal allowable radius of perturbations is given by $r=1 / S N_{\Delta, p}\left(G_{22}\right)$. One implication of the small gain theorem is the result that the structured norm is a lower bound on $G_{22}$,

$$
S N_{\Delta, p}\left(G_{22}\right) \leq\left\|G_{22}\right\|_{p}
$$

because if $\left\|\Delta^{\Pi}\right\|_{p}<1 /\left\|G_{22}\right\|_{p}$, then $\left(I-G_{22} \Delta^{\Pi}\right)^{-1}$ is $\ell_{p}$-stable. Therefore, if $S N_{\Delta, p}$ is $\ell_{p}$-norm bounded, then so is $G_{22}$.

\subsubsection{Stability for LTV, NTV, and NTI perturbations}

The monetary authority is assumed to choose the elements of $K$, that minimize the structured norm, $S N_{\Delta, p}, p=2$ or $p=\infty$. The elements of the transfer matrix, $G_{22}$, are linear, timeinvariant, and hence $\ell_{1}$-stable. This allows us to define a $n \times n$ matrix, $\hat{N}$, of $\ell_{1}$ norms of the 
elements of $G_{22}$,

$$
\hat{N}=\left(\begin{array}{ccc}
\left\|G_{22}^{11}\right\|_{1} & \cdots & \left\|G_{22}^{1 n}\right\|_{1} \\
\cdot & \cdot & \cdot \\
\cdot & \cdot & \cdot \\
\cdot & \cdot & \cdot \\
\left\|G_{22}^{n 1}\right\|_{1} & \cdots & \left\|G_{22}^{n n}\right\|_{1}
\end{array}\right)
$$

which must be approximated numerically.

Dahleh and Diaz-Bobillo (1995) show that for LTV, NTV, and NTI perturbations and bounded $\ell_{\infty}$-induced norms, $(p=\infty)$, it is necessary and sufficient that the spectral radius of $\hat{N}$ be smaller than the inverse of the radius of allowable perturbations, $\rho(\hat{N}) \leq 1 / r$, where $\rho(\hat{N})$ is defined as the largest stable root of $\hat{N}$. With bounded $\ell_{2}$-induced norms, $(p=2)$, the condition $\rho(\hat{N}) \leq 1 / r$ is sufficient. Further, since the stability conditions are the same for all three types of perturbations, the computations are greatly simplified, involving minimization of $\rho(\hat{N})$ over the elements of $K$ in all cases; and the maximal acceptable radius of perturbation becomes $1 / \hat{\rho}(\hat{N})$.

These three cases seem to be the most relevant ones for a monetary authority forming robust policy, given that $G_{22}$ is a function of the reduced-form solution of the parameters of a forward-looking structural model. If the authority is concerned about the about worst-case consequences of misspecification in some or all structural parameters, the effect on the reduced form will be nonlinear and will in general, though not always, involve most of its elements. In addition, by the Lucas critique, the elements of $\Pi$ may be time varying, so that, generally, parameter perturbations of any kind in the structural model may also translate into time-varying perturbations of the reduced form.

\subsubsection{Stability with LTI perturbations}

Linear time invariant perturbations can involve one-time parametric shifts or changes involving lags. The analysis is best carried out in the complex plane, which allows for an economical treatment of perturbations with infinite moving averages. The idea is the following. ${ }^{21}$ Assume we can model the $i j$-th diagonal component of the worst-case perturbation, $\Delta_{i j}^{\Pi}(L)$, obeying $\left\|\Delta_{i j}^{\Pi} / \delta_{i j}\right\|_{\infty}=r_{i j}<r$, with a very general infinite-order moving average, expressed as a

\footnotetext{
${ }^{21}$ We would like to thank Alexei Onatski for clarifying for us a number of points in the following discussion.
} 
polynomial on $L$,

$$
\begin{aligned}
\Delta_{i j}^{\Pi} / \delta_{i j} & = \pm r_{i j}\left(L-z_{i j}\right) /\left(1-z_{i j} L\right) \\
& = \pm r_{i j}\left[-z_{i j}+\left(1-z_{i j}^{2}\right) L+\left(1-z_{i j}^{2}\right) z_{i j} L^{2}+\cdots\right]
\end{aligned}
$$

where $z_{i j} \in(-1,1)$. Notice that for $z_{i j}=0$, the effect of a perturbation is via the $\pm r_{i j} L$ operator, so that the worst-case effect of a dynamic misspecification involves only one lag. Conversely, if $\left|z_{i j}\right|$ is close to 1 , all terms except the first are vanishingly small. Hence, this situation represents the case when the effect of the operator is not dynamic but scalar: $\pm r_{i j} z_{i j}$, such as a structural break. For values of $z_{i j}$ between 0 and $1, \Delta_{i j}^{\Pi}(L)$ represents a complex perturbation equivalent to an infinite-order polynomial in $L$ with rate of decay determined by $z_{i j} \cdot{ }^{22}$

In Zhou et al. (1996) it is shown that the linear time invariant perturbation in (24) satisfies,

$$
\Delta_{i j}^{\Pi}\left(\omega_{0}\right)=\delta_{i j} r_{i j} e^{i \omega_{i j}}
$$

where $i=\sqrt{-1}$, and $\omega_{0} \in[0, \pi)$. The following correspondence can be established between (24) and (25): the sign in (24) is positive if $\omega_{i j} \in[0, \pi)$, and it is negative if $\omega_{i j} \in(-\pi, 0)$. Hence, in both instances, $z_{i j}=\left(e^{i \omega_{0}}-e^{i \omega} /\left(1-e^{i\left(\omega_{0}+\omega_{i j}\right)}\right)\right.$. Therefore, by identifying $\omega_{0}$ and $r_{i j}$, it is possible to unravel the structure of $\Delta_{i j}^{\Pi}$.

The next step is to determine a feedback vector $K$ for which the important condition, $\left\|\Delta_{i j}^{\Pi} / \delta_{i j}\right\|_{\infty}=r_{i j}<r$, under which the preceding results hold, is, indeed, satisfied. The small gain theorem then guarantees that the system will be stable under the worst-case scenario.

Equation (25) can be evaluated numerically, again by exploiting the small gain theorem, this time in the frequency domain, to define a transfer function $\mathcal{G}_{22}$ eqivalent to $G_{22}$ above. Consider the augmented input output system (19)-(20). The transfer function from both disturbances, $v_{t}$, and perturbations, $h_{t}$, to the state, $x_{t}$, and the augmented state, $p_{t}$, is denoted $\mathcal{G}$ to distinguish it from $G$ :

$$
\begin{aligned}
{\left[\begin{array}{l}
x_{t} \\
p_{t}
\end{array}\right] } & =\left[\begin{array}{l}
I_{n} \\
\mathcal{U}
\end{array}\right]\left(I e^{\xi}-\Pi\right)^{-1}\left[\begin{array}{ll}
C & \Gamma
\end{array}\right]\left[\begin{array}{l}
v_{t} \\
h_{t}
\end{array}\right] \\
& \equiv\left[\begin{array}{ll}
\mathcal{G}_{11} & \mathcal{G}_{12} \\
\mathcal{G}_{21} & \mathcal{G}_{22}
\end{array}\right]\left[\begin{array}{l}
v_{t} \\
h_{t}
\end{array}\right]
\end{aligned}
$$

\footnotetext{
${ }^{22}$ For the numerical exercises, we found that the $z_{i j}$ 's, which we do not report, implied an average persistence with mean lag of about 3 quarters.
} 
To develop a notion of stability under LTI perturbations, we define yet another variation on structured norms, the structured singular value function. The feedback loop between $p_{t}$ and $h_{t}$, which links the perturbations, $\Delta^{\Pi}$, to the system variables, $x_{t}$, is $p_{t}=\mathcal{G}_{22} h_{t}$. For $p=2$ and $p=\infty$, the structured singular value function is then defined as,

$$
\mu_{\Delta \in L T I_{p}}\left[\mathcal{G}_{22}\right]=\frac{1}{\inf _{\Delta^{\Pi} \in L T I_{p}}\left\{\bar{\sigma}\left[\hat{\Delta}^{\Pi}\right] \mid \operatorname{det}\left(I-\mathcal{G}_{22} \Delta^{\Pi}\right)=0\right\}}=\inf _{\Delta^{\Pi} \in L T I_{p}} \rho\left(\mathcal{G}_{22} \Delta^{\Pi}\right)
$$

and if there is no $\Delta^{\Pi} \in D_{r}$ such that $\operatorname{det}\left(I-\mathcal{G}_{22} \Delta^{\Pi}\right)=0$, then $\mu_{\Delta^{\Pi} \in L T I}\left(\mathcal{G}_{22}\right)=0$.

The structured singular value function may be thought of as the frequency domain parallel to the structured norm function (22). The authority now chooses $K$ to minimize the structured singular value, $\mu$

$$
\mu=\inf _{K} S N_{\Delta_{L T V, p}}\left(\mathcal{G}_{22}\right)=\inf _{K} \sup _{\omega \in[0,2 \pi]} \mu_{\Delta_{L T I_{p}}}\left[\mathcal{G}_{22}\right] \leq \inf _{K} \sup _{\xi} \bar{\sigma}\left(\mathcal{G}_{22}\right),
$$

where $\sigma\left(\mathcal{G}_{22}\right)$ is the singular value of $\left(\mathcal{G}_{22}\right)$.

The maximum radius of allowable LTI perturbations is the inverse of $\mu$,

$$
\sup _{K} r=\frac{1}{\min _{K} \sup _{\omega \in[0,2 \pi]} \mu_{\Delta_{L T I_{p}}}\left[\mathcal{G}_{22}\right]} \text {. }
$$

\section{The model}

We seek a framework for policy that is simple, empirical, and realistic from the point of view of a monetary authority. Towards this objective, we construct a simple New Keynesian model along the lines of Fuhrer and Moore (1995b). The key to this model, as in any Keynesian model, is the price equation or Phillips curve. Our formulation is very much in the same style as the real wage contracting model of Fuhrer and Moore (1995a). By making use of the Fuhrer-Moore formulation, we 'slip the derivative' in the price equation, making inflation sticky and not just the price level, thereby ruling out the possibility of costless disinflation. However, instead of the fixed-term contract specification of Fuhrer-Moore, we adopt the stochastic contract duration formulation of Calvo. In doing this, we significantly reduce the state space of the model, thereby accelerating the numerical exercises that follow.

Equations (26) and (27) together comprise a forward-looking Phillips curve, with $\pi$ and $c$ measuring aggregate and core inflation, respectively, and $y$ is the output gap, a measure of excess demand. Equation (26) gives inflation as a weighted average of inherited inflation, $\pi_{t-1}$, and expected core inflation, $E_{t-1} c_{t+1}$. Following Calvo (1983), the expiration of contracts 
is given by an exponential distribution with hazard rate, $\delta$. Assuming that terminations of contracts are independent of one another, the proportion of contracts negotiated s periods ago that are still in force today is $(1-\delta) \delta^{t-s}$.

$$
\begin{aligned}
\pi_{t} & =\delta \pi_{t-1}+(1-\delta) E_{t-1} c_{t}+v_{t}^{\pi} \\
c_{t} & =(1-\delta) E_{t-1}\left(\pi_{t}+\gamma y_{t}\right)+\delta E_{t-1} c_{t+1} \\
y_{t} & =\phi_{1} y_{t-1}+\phi_{2} y_{t-2}+\phi_{3} R_{t-1}+v_{t}^{y} \\
R_{t} & =\frac{1}{1+D} E_{t-1} \sum_{i=0}^{\infty}\left(\frac{D}{1-D}\right)^{i}\left(r_{t+i}-\pi_{t+i}\right) \\
r_{t} & =g_{r} r_{t-1}+\left(1-g_{r}\right)\left(\bar{\pi}_{t}+r^{*}\right)+g_{\pi}\left(\bar{\pi}_{t}-\pi^{*}\right)+g_{y} y_{t}
\end{aligned}
$$

where it is assumed that the central bank reacts to the behavior of the average rate of inflation over the past year, where $\bar{\pi}=\frac{1}{4} \sum_{1}^{4} \pi_{t-i}$. In equation (27), core inflation is seen to be a weighted average of future core inflation and a markup of excess demand over inherited inflation. Equations (26)and (27) differ from the standard Calvo model only in that the dependent variables are rates of changes rather than levels. Equation (28) is a very simple aggregate demand equation with output being a function of two lags of output as well as the lagged $e x$ ante long-term real interest rate, $R$. Equation (29) follows Fuhrer and Moore (1995b) in using a constant approximation to duration formula by Macaulay (1938) defining the ex ante long-term real interest rate as a geometrically declining weighted average of current and future short-term real interest rates. Finally, equation (30) is a generic interest rate reaction function, written here simply to complete the model. We may assume that it is the empirical manifestation of an optimal decision rule by the Federal Reserve, which manipulates the nominal federal funds rate, $r$, and implicitly deviations of the real rate from its equilibrium level, $r^{*}-\pi^{*}$, with the aim of moving average annual inflation to its target level, $\pi^{*}$, reducing excess demand to zero, and penalizing movements in the instrument itself.

The model is stylized, but it does capture what we would take to be the fundamental aspects of models that are useful for the analysis of monetary policy. Among these, stickiness of inflation is foremost. Other integral features of the model include that policy acts on demand and prices with a lag. This rules out monetary policy that can instantaneously offset shocks as they occur. The model also assumes that disturbances to aggregate demand have persistent effects, as are the effects of demand itself on inflation. These features imply that in order to be effective, monetary policy must look ahead, setting the federal funds rate today to achieve objectives in the future. However, the stochastic nature of the economy implies that these plans will not be achieved on a period-by-period basis. Rather, the contingent plan set out by the authority in any one period will have to be updated as new information is revealed regarding 
the shocks that have been borne by the economy.

\subsection{The estimated model}

We estimated the key equations of the model on U.S. data from 1972Q1 to 1996Q4. ${ }^{23}$ Since the precise empirical estimates of the model are not fundamental to the issues examined here, we will keep our discussion of them concise. A couple of important points should be mentioned however. We measure goods-price inflation, $\pi$, with the quarterly change in the chain-weight GDP price index, a producer's price. However, we proxy $E_{t-1} c_{t+1}$ with the median of the Michigan survey of expected future inflation. The survey has some good features as a proxy. First, it is an unbiased predictor of future inflation. At the same time, it is not efficient: Other variables do help in predicting movements in future inflation. Second, it measures consumer price inflation expectations, precisely the rates that would theoretically go into wage bargaining decisions, and thereby into unit labor costs. GDP price inflation can then be thought of as a pseudo-mark-up over these expected future costs. The disadvantage is that the survey is for inflation over the next twelve months, which does not match the quarterly frequency of our model. However, most of the predictive power of the survey to predict inflation over the next twelve months comes from its ability to predict inflation in the very short term rather than later on, so this problem is not too serious.

Equation (27) can be substituted into equation (26) to yield a restricted Phillips curve. The estimates of this equation along with two others are presented in Table 1 below. Unemployment gaps-defined as the deviation of the demographically adjusted unemployment rate less the NAIRU-performed better in estimation than did output gaps, and so the former appears in equation (A) of the table. We then supplemented the empirical model with a simple Okun's Law relationship, equation (C), and then substituted it in order to arrive at the appropriate estimates for the equations (26) through (30).

The equation of primary interest is our Phillips curve. As equation (A) in the table shows, we supplemented the basic formulation with a small number of exogenous supply shock terms, including oil prices, a variable to capture the effects of the Nixon wage-and-price controls, and a constant term. These are traditional and uncontroversial inclusions. Roberts (1995) has found oil prices to be important for explaining inflation in estimation, using Michigan survey data.

The key parameters are the 'contract duration' parameter, $\hat{\delta}$, and the excess demand parameter, $\hat{\gamma}$. If this were a level contracts model, $\delta=.41$ would be an unreasonably low number since it implies a very short average contract length. For the present model, this interpretation

\footnotetext{
${ }^{23}$ The estimated interest reaction function is based on data ranging from 1980Q1 to 1998Q4 to reflect the now relevant policy regime spanned by the Volcker-Greenspan chairmanships of the Federal Reserve Board.
} 


\section{Table 2: Estimates of the Basic Contract Model (1972Q1 - 1996Q4)}

\begin{tabular}{|c|c|c|c|c|}
\hline \multicolumn{5}{|c|}{ A. $\pi=\left[1-(1-\delta)^{2}\right]^{-1}\left[\delta \pi_{t-1}+(1-\delta)^{2} \gamma u_{t-1}+(1-\delta) c_{t, t+1}\right]+\Gamma Z^{a b}$} \\
\hline Nixon price controls & $\hat{Z}_{1}$ & -5.35 & $(2.20)$ & $R^{2}=.97$ \\
\hline Change in oil prices & $\hat{Z}_{2}$ & 0.0019 & $(.70)$ & $\mathrm{SEE}=1.02$ \\
\hline Unemployment & $\hat{\gamma}$ & -0.23 & $(1.49)$ & $\mathrm{B}-\mathrm{G}(1)=.01$ \\
\hline Contract duration & $\hat{\delta}$ & 0.41 & $(4.65)$ & Constrained linear IV \\
\hline \multicolumn{5}{|c|}{ B. $y=\phi_{0}+\phi_{1} y_{t-1}+\phi_{2} y_{t-2}+\phi_{3} r l_{t-1}$} \\
\hline First lag output & $\phi_{1}$ & 1.22 & $(12.16)$ & $R^{2}=.88 \mathrm{SEE}=1.21$ \\
\hline Second lag output & $\phi_{2}$ & -0.36 & $(4.02)$ & $\mathrm{B}-\mathrm{G}(4)=0.05$ \\
\hline Real 5-year real rate & $\phi_{3}$ & -0.26 & $(2.41)$ & OLS \\
\hline \multicolumn{5}{|c|}{ C. $u=\gamma_{0}+\gamma_{1} y_{t}+\gamma_{2} T+\gamma_{3}\left(\right.$ poil $\left._{t} / p_{t}\right)$} \\
\hline Output gap & $\hat{\gamma}_{1}$ & -0.34 & $(9.01)$ & $R^{2}=0.80 \mathrm{SEE}=0.60$ \\
\hline Time trend & $\hat{\gamma}_{2}$ & 0.008 & $(1.81)$ & $\mathrm{B}-\mathrm{G}(4)=0.00$ \\
\hline Relative oil price & $\hat{\gamma}_{3}$ & 0.57 & $(3.07)$ & 2SLS \\
\hline \multicolumn{5}{|c|}{ D. $r=\left(1-g_{r}\right)\left(r^{*}-\pi^{*}\right)+g_{r} r_{t-1}+\left(1-g_{r}+g_{\pi}\right) \bar{\pi}_{t}+g_{y} y_{t}$} \\
\hline Inflation & $\left(1-g_{r}+g_{\pi}\right)$ & 0.324 & $(2.37)$ & $R^{2}=0.90 \mathrm{SEE}=1.23$ \\
\hline Output Gap & $g_{y}$ & 0.148 & $(2.22$ & $\mathrm{B}-\mathrm{G}(4)=0.00$ \\
\hline Interest Rate & $g_{r}$ & 0.803 & $(13.8)$ & Restricted OLS \\
\hline
\end{tabular}

${ }^{a}$ Data: change in oil prices is a four-quarter moving average of the price of oil imported into the U.S.; is the quarterly change at annual rates of the chain-weight GDP price index; is the demographically corrected unemployment rate, less the natural rate of unemployment from the FRB/US model database; is proxied by the median of the Michigan survey of expected inflation, 12 months ahead; is the output gap for the U.S. from the FRB/US model database; is the real interest rate defined as the quarterly average of the federal funds rate less a four-quarter moving average of the chainweight GDP price index; is the price of imported oil relative the GDP price index; and Nixon price controls equals unity in 1971Q4 and -0.6 in 1972Q1. All regressions also included an unreported constant term. Constants were never statistically significant. B-G(1) is the probability value of the Breusch-Godfrey test of first-order serial correlation.

${ }^{b}$ Notes: Equation (A) is estimated with instruments: constant, time trend, lagged unemployment gap, four lags of the change in imported oil prices; two lags of inflation, lagged real interest rate, lagged Nixon wage-price control dummy, and the lagged relative price of imported oil. Standard errors for all three equations were corrected for autocorrelated residuals of unspecified form using the Newey-West (1987) method. 
is not warranted, however. An estimate of $\delta=.41$ implies substantial persistence in inflation, much more so than any nominal wage contracting model could furnish. In fact, when equation (A) is solved, its reduced-form coefficient on lagged inflation is seen to be 0.846 . This is substantial inflation stickiness by any measure.

Turning to the aggregate demand function, it is conventionally believed that demand in the U.S. responds to movements in the long-term real interest rate. ${ }^{24}$ Accordingly, we define the ex ante real interest rate, $R_{t}$, as the five-year government bond rate less the average inflation rate that is expected over the next five years, and compute the latter using a small-scale vector autoregression. ${ }^{25}$. Five years is about the time period for which consumer durables and automobiles are typically financed. The duration, D, in equation (29), is set at 20 quarters in conformation with the definition of R.

The estimates of the aggregate demand function show the humped shape pattern of responses to output to demand shocks; that is, an exogenous disturbance to demand tends to overshoot initially-as determined by $\hat{\phi}_{1}=1.22>1$ - and then drop back, as indicated by $\hat{\phi}_{2}=-.36<0$. The interest elasticity of aggregate demand is large and negative as expected.

After substituting equation (C) into equation (A) and dropping those arguments that are not of interest to us, we arrive where we began: with equations (26) through (30). The parameters of the estimated model are broadly similar to estimates of other models, and are reasonable. Impulse responses of the model to exogenous shocks to the price equation and the aggregate demand function are consistent with the historical experience in the U.S. as measured by simple vector autoregressions. The estimated equations do show some remaining residual correlation. This is a common finding in structural price equations-Roberts (1995) uncovered the same phenomenon-so as noted above, we have corrected the variance-covariance matrix for this autocorrelation using the Newey and West (1987) technique. We conclude that our model is appropriate for the questions we wish to address.

\section{Robust Policy Exercises}

The monetary policy authority's task is to keep inflation, $\pi$, close to its target level, taken without loss of generality to be zero, and to do so at minimum cost in terms of output losses, $y$, defined as the percent deviation of GDP from trend. We also include a third target variable,

\footnotetext{
${ }^{24}$ See Fuhrer and Moore (1995a) for an extensive discussion of the linkage between monetary policy and the long-term interest rate.

${ }^{25}$ This is the same methodology as employed in the FRB/US macroeconomic model of the US, built and maintained by the Federal Reserve Board. For more information, see Brayton and Tinsley (1996) and Brayton, Mauskopf, Reifschneider, Tinsley and Williams (1997)
} 
the change in the short-term interest rate, $\Delta r_{t}$, because in the present model, as in most other models, the parameters in the interest rate reaction function are absurdly large in the absence of some penalty on instrument variability.

The model is implicitly derived from first-order conditions determined by optimizing agents who form rational expectations by solving the model, which they assume to be true. In contrast, the central bank is assumed to have doubts about that model as a true representation of the economy. ${ }^{26}$

In terms of the generic model outlined in Section 2.1, the state vector of the preceding model is defined by, $x_{t+1}=\left[y_{t}, \pi_{t}, r_{t}, y_{t-1}, \pi_{t-1}, r_{t-1}, \pi_{t-2}, \pi_{t-3}, c_{t+1}, R_{t+1}\right]^{\prime}$. The instrument is $r_{t}$, and the target vector is, $T_{t}=\left[y_{t}, \pi_{t}, \Delta r_{t}\right]^{\prime}$. The selector matrices for the mapping from states to targets in (2) and the weighting matrix $Q$, are

$$
M_{x}=\left[\begin{array}{cccccccccc}
1 & 0 & 0 & 0 & 0 & 0 & 0 & 0 & 0 & 0 \\
0 & 1 & 0 & 0 & 0 & 0 & 0 & 0 & 0 & 0 \\
0 & 0 & 0 & 0 & 0 & -1 & 0 & 0 & 0 & 0
\end{array}\right], M_{u}=\left[\begin{array}{l}
0 \\
0 \\
1
\end{array}\right], Q=\left[\begin{array}{ccc}
\sqrt{\gamma_{y}} & 0 & 0 \\
0 & \sqrt{\gamma_{\pi}} & 0 \\
0 & 0 & \sqrt{\gamma_{\Delta r}}
\end{array}\right]
$$

We will distinguish two cases of preference orderings over inflation and output performance: (1) a central bank with "strong" inflation preferences, i.e., one that puts relatively more emphasis on controlling inflation, and (2) a central bank with "weak" inflation preferences, i.e., one that puts relatively more emphasis on controlling output. Formally, the preference parameters are,

Strong: $\gamma_{y}=.2, \gamma_{\pi}=.8$, and $\gamma_{\Delta r}=.01 ;$ Weak $: \gamma_{y}=.8, \gamma_{\pi}=.2$, and $\gamma_{\Delta r}=.01$.

In each case, a miniscule weight is placed on interest rate changes. The optimal control literature as well as most papers on robust control traditionally place emphasis on synthesizing fully parameterized feedback rules. Given the strong interest in simple, Taylor-style interest rate rules and the impracticality of optimal control solutions with large models, we shall restrict our attention to simple robust rules that respond to the previous period's movements in only three state variables, the output gap, $y$, the inflation rate, $\pi$, and the federal funds rate, $r$. Obviously, this restriction imposes an additional burden of choosing a suitable subset of the state variables, an issue that is still under debate as the growing literature on simple monetary policy rules demonstrates. Experience suggests that the cost of such parsimony appears to be negligible. For example, Tetlow and von zur Muehlen (1996) show that for a model very similar to the one used here, the differences in welfare loss resulting from optimal control and

\footnotetext{
${ }^{26} \mathrm{We}$ assume that, while the monetary authority is risk-sensitive, agents in the economy believe the reference model to be true. Hansen and Sargent (1999b) assume that agents and the authority share the same preference for robustness, indexed by a single parmater, $\theta$.
} 
optimal simple rules are small.

The parameter vector, $K$, of the policy rule introduced in section 2.1 becomes $K=$ $\left[g_{r}, g_{\pi}, g_{y}\right]$, multiplying the lagged values of the federal funds rate, the inflation rate, and the output gap, respectively.

Recall that in section 2, we defined five optimization problems, three associated with unstructured uncertainty, and two with structured uncertainty. Table 3 serves as a reminder of the methods utilized in what follows. Under the heading "Unstructured Uncertainty," the authority chooses a feedback rule, $u_{t}=K x_{t}$ that minimizes the three loss functions $\mathcal{H}^{2}, \mathcal{H}^{\infty}$, and $\ell_{1}$. Under the heading "Structured Uncertainty," the authority chooses $K$ that maximizes the radius, $\hat{r}$, of allowable perturbations, as previously defined.

Table 3: Criteria for Robust Policies

\begin{tabular}{c||c|c}
\hline \multicolumn{1}{c||}{ Criteria for } & \multicolumn{2}{c}{ Criteria for } \\
Unstructured Uncertainty & \multicolumn{2}{c}{ Structured Uncertainty } \\
& NTV & LTI \\
\hline \hline $\min \mathcal{H}^{2}, \min \mathcal{H}^{\infty}, \min \ell_{1}$ & $\hat{r}=\max \frac{1}{\hat{\rho}(\hat{N})}$ & $\hat{r}=\max \frac{1}{\hat{\mu}\left(\mathcal{G}_{22}\right)}$ \\
\hline \hline
\end{tabular}

We consider two ways of looking at the consequences of applying the various robust policy rules derived here to the estimated model. In each case, we compare the derived rule with the estimated rule or the optimal (restricted) linear-quadratic rule.

The first and most obvious measures of performance are the rules themselves, their implications for the steady state, and the implied losses. In the case of structured model uncertainty, the allowable radius of perturbations (the inverse of the minimum structured norm) gives a measure of the size of maximum uncertainty the authority is capable of tolerating. Since in most cases, this may be too strict a criterion, a preferred interpretation of the "maximum of allowable perturbations" as an upper bound. A wider range of policies becomes available if the central bank considers the opponent to be less malevolent, that is, if uncertainty can be bounded in a smaller set.

A second way to view the implications of various policies is to evaluate $\mathcal{H}^{2}$ welfare losses at various frequencies, making use of the definition in (14). For example, while the optimal linearquadratic rule performs well for serially uncorrelated shocks that give rise to cycles at quarterly to 2-year frequencies, it turns out that it leaves policy vulnerable to small mispecifications of the temporal and feedback properties of shocks affecting business cycle frequencies. A surprising 
finding is that this is particularly true of historic policy as represented by our estimated rule and filtered through the model.

\subsection{Unstructured model uncertainty}

The sets of rules that stabilize performance of the target variables $y, \pi$, and in a minor way, $\Delta r$, were calculated for $\mathcal{H}^{2}, \mathcal{H}^{\infty}, \ell_{1}$ losses. We omit minimum entropy results since it is clear that they constitute intermediate outcomes between $\mathcal{H}^{2}$ and $\mathcal{H}^{\infty}$ policies.

Tables 4 and 5 show the detailed results for "strong" and "weak" anti-inflation preferences, respectively. The left-hand column indicates the norms imposed on the loss function and uncertainty; the next column to the right shows the induced norm, i.e., the indirect loss function implied by the first column; the next three columns present the impact coefficients of the calculated optimal rule, and the following two columns present the implied equilibrium reactions to inflation and output; the largest stable roots are listed under "spectral radius." The last column gives the implied $\mathcal{H}^{2}$ losses, which measure the welfare loss under the original LQR criterion. This measures how much performance the authority gives up if it is truly paranoid and the only shocks the economy experiences are those of the estimated variance covariance matrix. This column shows that robustness against worst-case outcomes can only be achieved at the expense of average performance. Interestingly, the estimated rule generates the highest $\mathcal{H}^{2}$ loss.

\section{The $\mathcal{H}^{2}$ LQR rule,}

Both tables show that the LQR rule, while less autoregressive than the estimated rule, has significantly larger reaction coefficients for inflation and output than the estimated rule. This now familiar result has been the spark for much of recent writing on policy timidity, including the present paper.

\section{The $\mathcal{H}^{\infty}$ robust rule}

The results for $\mathcal{H}^{\infty}$ policies are a bit more subtle and depend on the assumed inflation fighting attitude of the authority. A "strong" inflation fighter practices a policy of anti-attenuation, reacting to output fluctuations and especially discrepancies of inflation from target with gusto. The "weak" inflation targeting authority is more circumspect: Its policy rule is much more comparable to the certainty equivalent rule. In addition, the "weak" authority tends to show a substantially greater degree of persistence-or instrument smoothing — in its setting of the funds rate, as shown by the large coefficient on the lagged funds rate term in the rule. This too, is much like the certainty-equivalent (LQR) rule. The key to understanding this result is recognizing that output control appears earlier in the monetary policy transmission mechanism, and that it is governed by an $\mathrm{AR}(2)$ process: Control of output operates more-or-less directly-but 
with persistence - while control of inflation, to a first approximation, must be carried out indirectly through manipulating output. An authority that cares relatively a great deal about output will, in the presence of large and persistent (perceived) shocks tend to match that persistence, using the persistence in output itself, along with persistent movements in the funds rate. That this may produce poor outcomes in terms of inflation is less of a worry since inflation carries a low weight in the loss function. A "strong" inflation fighting central bank, by contrast, will tend to aggressively adjust the funds rate to move output, and work against the persistence in output, to attack inflation any time it deviates from target.

It is worth noting that the results reported here for robust control with unstructured uncertainty echo the results in previous research. Sargent (1999), for example, finds that robust rules applied to the backward-looking open-economy model of Ball (1998) are more aggressive than the optimal LQR rule, and become increasingly aggressive as risk sensitivity approachs that implied by an optimal $\mathcal{H}^{\infty}$ solution. Similarly, Onatski and Stock (2000), find that $\ell_{1}$ and $\mathcal{H}^{\infty}$ criteria produce more aggressive feedback rules in the Rudebusch and Svensson (1999) backward-looking closed-economy model of the U.S. economy.

Table 4: Rules under Shock and Model Uncertainty:"Strong” Inflation Preference

\begin{tabular}{|c|c|c|c|c|c|c|c|c|}
\hline \multirow{2}{*}{$\begin{array}{c}\text { Bounds } \\
\text { Loss/Perturbation }\end{array}$} & \multirow{2}{*}{$\begin{array}{l}\text { Induced } \\
\text { Norm }\end{array}$} & \multicolumn{5}{|c|}{ Parameters in the Rule } & \multirow{3}{*}{$\begin{array}{c}\text { Spectral } \\
\text { Spectral Radius }\end{array}$} & \multirow{2}{*}{$\begin{array}{l}\text { Implied } \\
\mathcal{H}^{2} \text { Loss }\end{array}$} \\
\hline & & \multicolumn{3}{|c|}{ Impact } & \multicolumn{2}{|c|}{ Equilibrium } & & \\
\hline & & $r$ & $\overline{\bar{\pi}}$ & $y$ & $\overline{\bar{\pi}}$ & $y$ & & \\
\hline LQR & $\mathcal{H}^{2}$ & 0.73 & 4.24 & 1.81 & 16.7 & 6.7 & 0.82 & 3.8 \\
\hline$\ell_{2}$ loss $/ \ell_{2}$ shock & $\mathcal{H}^{\infty}$ & 0.25 & 16.6 & 8.5 & 23.1 & 11.3 & 0.65 & 5.0 \\
\hline$\ell_{\infty}$ loss $/ \ell_{\infty}$ shock & $\ell_{1}$ & 0.53 & 9.60 & 5.23 & 21.4 & 11.2 & 0.73 & 4.2 \\
\hline Estimated Rule & & 0.80 & 0.32 & 0.15 & 2.6 & 0.8 & 0.88 & 5.8 \\
\hline
\end{tabular}

Table 5: Rules under Shock and Model Uncertainty: "Weak" Inflation Preference

\begin{tabular}{|c|c|c|c|c|c|c|c|c|}
\hline \multirow{2}{*}{$\begin{array}{c}\text { Bounds } \\
\text { Loss/Perturbation } \\
\end{array}$} & \multirow{2}{*}{$\begin{array}{c}\text { Induced } \\
\text { Norm }\end{array}$} & \multicolumn{5}{|c|}{ Parameters in the Rule } & \multirow{2}{*}{$\begin{array}{c}\text { Spectral } \\
\text { Spectral Radius } \\
\end{array}$} & \multirow{2}{*}{$\begin{array}{l}\text { Implied } \\
\mathcal{H}^{2} \text { Loss } \\
\end{array}$} \\
\hline & & \multicolumn{3}{|c|}{ Impact } & \multicolumn{2}{|c|}{ Equilibrium } & & \\
\hline & & $r$ & $\bar{\pi}$ & $y$ & $\bar{\pi}$ & $y$ & & \\
\hline LQR & $\mathcal{H}^{2}$ & 0.66 & 1.89 & 3.51 & 6.6 & 10.3 & 0.79 & 3.8 \\
\hline$\ell_{2}$ loss $/ \ell_{2}$ shock & $\mathcal{H}^{\infty}$ & 0.70 & 2.10 & 2.35 & 8.0 & 7.8 & 0.76 & 5.0 \\
\hline$\ell_{\infty}$ loss $/ \ell_{\infty}$ shock & $\ell_{1}$ & 0.25 & 5.88 & 16.4 & 8.8 & 21.9 & 0.84 & 6.6 \\
\hline Estimated Rule & & 0.80 & 0.32 & 0.15 & 2.6 & 0.8 & 0.88 & 7.6 \\
\hline
\end{tabular}

\section{The $\ell_{1}$ (MAD) robust rule}

Under the $\ell_{1}$ loss criterion, the authority minimizes absolute target deviations. As stated earlier, this criterion is the induced loss function when the authority minimizes the maximum absolute value of target deviations, $\|z\|_{\infty}$, against the largest possible shock satisfying $\|w\|_{\infty}<\infty$. 
The tables show that MAD policies are universally more reactive than the LQR rules and less aggressive than $\mathcal{H}^{\infty}$ policies under strong inflation preferences than under weak inflation preferences, both with respect to autoregressivity of the interest-rate rule and the size of impact reaction parameters. In comparing these two policies, note that under $\mathcal{H}^{\infty}$ control, the decision maker protects the average square metric of performance against an average square metric of misspecification errors, while in a MAD rule, the attempt is to guard against outlier outcomes. Given the character of the estimated policy channel from interest rate movements to output and inflation in this model, it appears that a strong preference for preventing economic booms or busts produces faster and stronger responses to signals from the economy than a concern for smooth economic behavior.

\section{Frequency decompositions of losses}

To illuminate how preference for robustness manifests itself in the potential performance of the authority's goals, it is useful to plot the $\mathcal{H}^{2}$ losses implied by the four alternative rules. ${ }^{27}$ Figure 1 shows this for the strong inflation targeting authority. The curves capture how different attitudes toward robustness affect average performance, under the assumption that the reference model is correct. A relatively flat curve suggests that the authority has managed to insulate itself against shocks in a broad range of frequencies, while a curve that has a peak-power concentrated within a narrow frequency band-indicates that the authority is vulnerable to shocks affecting those frequencies. By assumption, nature could choose to concentrate its choice of shocks to attack that range

The LQR rule, which, by definition has the smallest area beneath it, has a pronounced peak at a four-year frequency. By contrast, the $\mathcal{H}^{\infty}$ and MAD policies tend to more evenly dispersed over all frequencies. As might be expected, the MAD rule, being the most risk sensitive, is the flattest, followed by the $\mathcal{H}^{\infty}$ rule. Under these criteria, the authority surrenders some average performance by increasing the area under the curve in order to minimize the maximum loss-in other words, the peak.

In contrast to the risk sensitive policies just discussed, which tend to immunize losses against adverse outcomes concentrated at particular frequencies, the estimated rule implies $\mathcal{H}^{2}$ losses that are strongly clustered around eight years, leaving the policy maker most vulnerable at typical business cycle frequencies.

\footnotetext{
${ }^{27}$ Technically, the plots show trace $\left[\mathcal{G}^{\prime}(\xi) \mathcal{G}(\xi)\right]$, which measures the $\mathcal{H}^{2}$ loss at frequency $\omega . \mathcal{G}(\xi)$ measures the amplification of shocks as measured by the performance of the loss function in frequency space. The horizontal axis displays quarters, and the vertical axis shows the value of the loss.
} 


\subsection{Structured model uncertainty}

In this section, we offer answers to questions like: "what should robust policy be if the central bank fears misspecification of a particular parameter, such as the slope of the Phillips curve?"28 or, "what is the best policy against losses arising from worst-case consequences of misspecified dynamics?" Misspecified or unmodelled dynamics are perhaps the most common sources of misspecification, given the difficulty of fully capturing all lagged effects in typical economic models.

As indicated in Section 2.2.2, we work with the reduced form based on the structural model, given a specification of the policy rule. In our case, it turns out that the top row of the reducedform matrix, $\Pi$, reproduces the structural IS equation with zero elements corresponding to all other state variables not specified in (28). This means that the reduced-form parameters for the IS curve are independent of the parameters in the remainder of the model. Any presumed parameter uncertainty in the remainder of the model has no effect on the uncertainty involving the IS curve parameters. The remaining parameters in $\Pi$ are nonlinear functions of all other parameters in the structural model, so that perturbations to any parameter in the structural model will affect parameters in all but the first row of the reduced form, with the exception of the own parameters in the IS curve: a perturbation to the slope of the IS curve, for example, affects only that parameter in the IS curve and all the elements in the second to eleventh rows of $\Pi$.

The exercises, while not specifically directed at perturbing structural parameters (as they would be in a backward looking model), can be interpreted as such in ways to be spelled out presently. Four interesting cases can be distinguished: (1) perturbations to all parameters, (2) perturbations to lagged output parameters, (3) perturbations to the slope of the IS curve, and (4) perturbations to the slope of the Phillips curve. As outlined in the theoretical sections, we distinguish three types of model perturbations. The LTI-scalar case is most restrictive, limiting uncertainty to very simple events, such as one-time structural breaks in one or more parameters. Nonlinear time-varying (NTV) perturbations reflect the most general case of model structure uncertainty. An intermediate case is LTI-MA, which assumes that the approximation errors of selected parameters can be modeled as infinite moving averages representing misspecified lag structures in the model. The results for these cases are summarized in Table $6 .^{29}$

\footnotetext{
${ }^{28}$ The source of misspecification of the slope of the Phillips curve, $(1-\delta)^{2} \gamma$, may be mismeasurement of the output gap. We may decompose $y$ as $y=\log Y-\log \hat{Y}-\epsilon_{y}$, where $Y$ and $\hat{Y}$ are the levels of actual and capacity output, respectively, and $\epsilon_{y}$ is the measurement error of capacity output. Thus, if one cannot distinguish between a shock to $y$ and a shock to $(1-\delta)^{2} \gamma$, then uncertainty about measurement is reflected in uncertainty about the slope. As noted in the introduction, Orphanides, Porter, Reifschneider, Tetlow and Finan (2000) find that output gap uncertainty leads to attenuation of interest rate reactions if the monetary authority minimizes Bayesian risk.

${ }^{29}$ The relevant augmented output vector (see equation (19)) is $p_{t}=\left[\pi_{t-1}, r_{t-1}, y_{t-1}, \pi_{t-2}, \pi_{t-3}, r r l_{t}\right]^{\prime}$, and $\mathcal{U}$
} 
Table 6: Interest Rate Rules with Structured Model Uncertainty

\begin{tabular}{|c|c|c|c|c|c|c|c|c|c|c|}
\hline \multicolumn{11}{|c|}{ Parameters in the Rule } \\
\hline & \multirow{3}{*}{\multicolumn{2}{|c|}{$\begin{array}{c}\text { Type of } \\
\text { Perturbation }\end{array}$}} & $(1)$ & (2) & (3) & (4) & $(5)$ & (6) & (7) & $(8)$ \\
\hline & & & \multicolumn{3}{|c|}{ Impact } & \multicolumn{2}{|c|}{ Equilibrium } & \multirow{2}{*}{$\begin{array}{c}\text { Spectral Radius } \\
\bar{\rho}\end{array}$} & \multirow{2}{*}{$\begin{array}{c}\text { Radius of } \\
\text { Perturbations }^{a}\end{array}$} & \multirow{2}{*}{$\begin{array}{l}\text { Implied } \\
\mathcal{H}^{2} \operatorname{Loss}^{L}\end{array}$} \\
\hline & & & $r$ & $\bar{\pi}$ & $y$ & $\bar{\pi}$ & $y$ & & & \\
\hline \multicolumn{11}{|c|}{ Perturbations to All Model Parameters } \\
\hline (1) & LTI & scalar & 0.05 & 1.05 & -1.30 & 2.1 & -1.4 & 0.89 & 0.24 & 17.3 \\
\hline (2) & LTI & MA & 0.92 & 0.08 & 0.39 & 1.1 & 4.8 & 0.88 & 0.21 & 13.6 \\
\hline (3) & NTV & & 0.65 & 0.74 & 0.78 & 3.1 & 2.2 & 0.76 & 0.15 & 6.6 \\
\hline \multicolumn{11}{|c|}{ Perturbations to lagged output parameters $\left(\phi_{1}, \phi_{2}\right)$} \\
\hline (4) & LTI & scalar & 0.84 & 0.16 & 0.12 & 2.0 & 0.8 & 0.90 & 0.31 & 10.8 \\
\hline (5) & LTI & MA & 0.93 & 0.07 & 0.38 & 2.0 & 5.4 & 0.89 & 0.22 & 14.9 \\
\hline (6) & NTV & & 0.65 & 0.78 & 0.82 & 3.2 & 2.3 & 0.76 & 0.16 & 6.4 \\
\hline \multicolumn{11}{|c|}{ Perturbations to slope of IS curve $\left(\phi_{3}\right)$} \\
\hline (7) & LTI & scalar & 0.44 & 1.24 & 0.65 & 3.2 & 1.1 & 0.77 & 0.22 & 4.9 \\
\hline (8) & LTI & MA & -0.08 & 1.08 & -0.48 & 2.0 & -0.4 & 0.86 & 0.23 & 11.4 \\
\hline (9) & NTV & & 0.64 & 0.73 & 0.71 & 3.2 & 2.0 & 0.76 & 0.19 & 4.8 \\
\hline \multicolumn{11}{|c|}{ Perturbations to slope of Phillips curve $(\delta, \gamma)$} \\
\hline (10) & LTI & scalar & -.97 & 1.97 & -2.29 & 2.0 & -1.2 & 0.97 & 0.39 & 13.8 \\
\hline (11) & LTI & MA & -0.98 & 1.98 & -0.91 & 2.0 & -0.5 & 0.98 & 0.24 & 13.0 \\
\hline (12) & NTV & & 0.58 & 0.83 & 0.70 & 3.0 & 1.7 & 0.75 & 0.20 & 4.9 \\
\hline$\overline{(\overline{(13)}}$ & & & 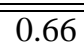 & $\overline{\overline{11.89}}$ & $\bar{~} \overline{3.51}$ & 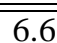 & $\overline{10.3}$ & $\overline{\overline{0.79}}$ & & $\overline{c 3.8}$ \\
\hline (14) & Estim & ed Rule & 0.80 & 0.32 & 0.15 & 2.6 & 0.8 & 0.88 & & 7.6 \\
\hline
\end{tabular}

${ }^{a}$ The radius of allowable perturbations is the inverse of the imputed $\ell_{\infty}$ loss.

${ }^{b}$ Evaluated for weak inflation targeting preference.

is chosen appropriately. The calculations for this section assume that perturbations to the structural parameters lie approximately within two standard deviations of the estimates. 
To jump to the punchline, as shown in Table 6, in contrast to robust policy against combined model and shock uncertainty, rules that are robust to structured model uncertainty are less aggressive than optimal LQR rules, in some cases approaching the estimated rule to a remarkable degree. A convenient measure of the relative aggressiveness of policy is the set of equilibrium response coefficients exhibited in columns 4 and 5. Compared with the LQR response to inflation of 6.6 , shown in row 13, the inflation responses of the robust rules are considerably weaker, ranging from 1.1 to 3.2. A similar conclusion holds for responses to the output gap. By contrast, the equilibrium responses of the robust rules are much more closely aligned with the estimated rule (row 14), especially in the case of the scalar LTI perturbations to the lagged output coefficients of the aggregate demand function in row 4. In general, there appears to be a rough hierarchy from more to less aggressive, depending on how much structure is imposed on model uncertainty. Policies based on the greatest amount of structure, the LTI rules, are the most attenuated, while policies based on the least amount of structure, the NTV rules, are the most aggressive. ${ }^{30}$ This finding allows us to put into context our earlier findings on attenuation with unstructured uncertainty with the Bayesian approaches surveyed above: it appears that the denser the fog of Knightian uncertainty, the greater the tendency for decisive action. Smaller and more localized uncertainties bring about cautious behavior, while more generalized ambiguities elicit stronger responses.

\section{Structural breaks}

The rows marked "LTI scalar," show robust rules when the authority attempts to avoid worst-case outcomes resulting from one-time permanent structural breaks in either the entire model, or in selected parameters. In each of these cases, policies become substantially attenuated; that is, the coefficients are uniformly lower than the LQR coefficients displayed in row 13 of the table. In particular, robust rules that protect against breaks in the parameters of the aggregate demand function-rows 4 and 7-give equilibrium rule parameters that are very close to the estimated rule. The same result applies for breaks in the slope of the Phillips curve (row 10), and in all model parameters simultaneously (row 1) - but only for inflation coefficients: One-time perturbations to the slope of the Phillips curve shown in row 10 lead to "perverse" responses to output owing to the possible breakdown of the normal lead-lag relationship between output and future inflation.

\section{Misspecified or unmodeled dynamics}

Policies that are sensitive to misspecified or unmodeled dynamics are represented by linear

\footnotetext{
${ }^{30}$ This conforms with Onatski and Stock (2000), who also find optimal policies becoming less aggressive as more structure is placed on uncertainty.
} 
time-invariant moving-average (LTI-MA) perturbations to any or all parameters in the model (rows 2, 5, 8 and 11). In general, the LTI-MA rules produce equilibrium responses to inflation that are close to that of the estimated rule, but with wide variations in equilibrium output gap coefficients. Considering, once again, the case of perturbations to the persistence parameters of the aggregate demand function, $\phi_{1}$ and $\phi_{2}$, shown in row 5, we see that the resulting policy also comes close to the estimated rule, although it produces too much persistence and too much aggressiveness in response to the output gap.

\section{Nonlinear time-varying perturbations}

The most noteworthy thing about the NTV rules shown in Table 6 (rows 3, 6, 9 and 12) is how similar they are notwithstanding the different origins of the perturbations considered. All the rules have impact coefficients on output and inflation of about 0.7 or so, and a coefficient on the lagged federal funds rate of about 0.6. Moreover, the implied equilibrium coefficients are all quite close to those of the estimated rule, particularly for inflation. However, the computed impact coefficients are too large, and persistence parameters are too small, to match the estimated rule.

Both the similarity of the rules across cases and the lack of persistence are manifestations of two aspects of the model uncertainty that are assumed: The perturbations have very little temporal stability given their non-linear and time-varying nature, and no persistence. The former implies that the origins of the shocks are of little use for the design of policy-resulting in a homogeneity of policy design. The latter suggests that without persistence in disturbances, there is little benefit to persistence in policy.

\section{Robustness and its costs}

Before leaving this topic, it is worth reflecting on the extent to which the policies in Table 6 protect against large shocks, and the costs of this protection. The degree of protection is measured by the radius of perturbations shown in column 7. The higher the number in the table, the larger the perturbations that the applicable rule can withstand. Not surprisingly, there is a tendency that the broader the scope of shocks the authority wants to protect againstsuch as in the NTV cases - the smaller the shock that can be protected against: Coverage against LTI-scalar shocks is shown to be greater than coverage against NTV shocks. Column 8 , however, shows the cost of this protection in those instances where the reference model turns out to be true; that is, when the economy actually faces only the estimated shocks. This column of the table demonstrates that in most cases, the protection against one-time structural shifts comes at a substantial price. This contrasts with the NTV cases where losses are uniformly lower. Evidently, a tinge of apprehension about generalized misspecification comes at a low 
cost relative to strong anxiety about uncertainty of a specific type.

\section{Frequency decomposition of losses}

Finally, we turn to the decomposition of losses, analogous to those of Figure 1, discussed previously. Figures 3 and 5 show the susceptibility of the rules discussed in Table 6 to perturbations to all parameters simultaneously, and to the lagged output parameters. These are the cases that span the shocks to model persistence. Notice that the LTI-scalar and especially the LTI-MA rules have patterns that closely match those of estimated rule. This observation confirms the impression left by Table 6 that the estimated rule may have been the outcome of efforts to protect against misspecified output persistence. Broadly similar, if less pronounced, results are obtained for the other sources of perturbations shown in Figures 7 and 9.

The most dramatic conclusion for robust policy under structured model uncertainty is that almost as a general rule, the estimated rule and the policies that best mimic it, are the most vulnerable to disturbances that produce phenomena at business cycle frequencies. Arguably, these are just the sort of shocks that central bankers would most often worry about. The exception are the NTV perturbations shown as the dotted lines in the figures. Taken at face value, these observations suggest that if central banks can overcome estimation problems with respect to such things as one-time breaks in trends, the remaining small but exotic perturbations can be handled well with rules that are robust, plausible, and protect against phenomena at business cycle frequencies. Moreover such rules are not costly to implement in terms of foregone performance in worlds where the reference model is true.

\section{Concluding Remarks}

We began this paper by reflecting on a puzzle: if monetary policy seeks to minimize output and inflation fluctuations, how does one explain the fact that historical interest-rate responses to these two indicators have been far more muted than suggested by optimal policy rules?

We have found, as others have, that optimal linear-quadratic rules derived in the absence of model uncertainty are indeed more reactive than rules estimated on data for the United States.

Stabilizing a monetary economy is a difficult job. The authority has but one instrument and usually at least two targets. The instrument works with a lag. Moreover, the authority faces an economy that is constantly changing, resulting in profound uncertainties regarding estimated structural parameters. Can such uncertainties explain the observed attenuation of policy? Our results suggest that the answer is yes and no.

We did find rules that protect against a class of specification errors, modeled as structured perturbations to a reference model, that resemble the estimated rule. However, we also found 
that robust policy rules that seek to guard against very general forms of misspecifications are even more reactive than the linear-quadratic rule. The robust rule that comes closest to approximating the estimated rule is one that seeks to guarantee a minimum level of stability against worst-case specification errors in the dynamics of aggregate demand. It follows that one possible interpretation of Fed behavior of the last twenty years is that the observed attenuation in policy was motivated by distrust regarding the estimated degree of output persistence. This motivation arises in large part because the aggregate demand function determines the dominant root—and hence the stability—of the model.

Given that the aggregate demand function is specified in terms of excess demand-meaning output relative to potential output - the literature on mismeasurment of potential output is of pertinence here. Work by Orphanides (1998), Smets (1999), Orphanides et al. (2000), and Tetlow (2000), among others, shows that potential output can be badly mismeasured and that correcting the measurement error can take a long time. Such errors could easily show up as mismeasured persistence in an aggregated demand function.

But if uncertainty of a particular structure can explain observed Fed behavior, what can be said about more generalized uncertainty? Our results suggest a hierarchy of policy responses measured in terms of attenuation or anti-attenuation indexed against the assumed degree of structure in Knightian uncertainty: The greater the structure on the uncertainty, the more likely policy attenuation is likely to arise. At the same time however, the more structure is assumed in the perturbations the authority faces, the larger the losses that are borne if the robustness turns out to have been unnecessary. 


\section{References}

Anderson, G., Moore, G., 1985, A linear algebraic procedure for solving linear perfect foresight models, Economics Letters 17, 247-252.

Anderson, G. S., 2000, A reliable and computationally efficient algorithm for imposing the saddlepoint property in dynamic models, Federal Reserve Board Finance and Economics Discussion Series forthcoming.

Aoki, M. 1967, Optimization of stochastic systems, Academic Press, New York.

Ball, L. 1998, Policy rules for open economies, in: J. B. Taylor ed., Monetary policy rules, U(niversity of Chicago Press, Chicago), 127-144.

Basar, T., Bernhard, P. 1991, $\mathcal{H}^{\infty}$-optimal control and related minimax design problems, (Birkäuser, Boston-Basel-Berlin).

Blinder, A. 1998, Central banking in theory and practice, (MIT Press, Cambridge).

Brainard, W., 1967, Uncertainty and the effectiveness of policy, American Economic Review $58,411-425$.

Brayton, F., Mauskopf, E., Reifschneider, D., Tinsley, P., Williams, J., 1997, The role of expectations in the FRB/US model, Federal Reserve Bulletin 83, 227-245.

Brayton, F., Tinsley, P. A., 1996, A guide to FRB/US: a macroeconomic model of the United States, Federal Reserve Board Finance and Economics Discussion Series 1996-42.

Calvo, G. A., 1983, Staggered prices in a utility-maximizing framework, Journal of Monetary Economics 12, 383-398.

Caravani, P., 1995, On $\mathcal{H}^{\infty}$ criteria for macroeconomic policy evaluation, Journal of Economic Dynamics and Control 19, 961-84.

Caravani, P., Papavassilopoulos, G., 1990, A class of risk-sensitive noncooperative games, Journal of Economic Dynamics and Control 14, 117-149.

Chow, G. C. 1975, Analysis and control of dynamic economic systems, (John Wiley and Sons, New York).

Craine, R., 1979, Optimal monetary policy with uncertainty, Journal of Economic Dynamics and Control 1, 58-93. 
Dahleh, M. A., Diaz-Bobillo, I. J. 1995, Control of uncertain systems: a linear programming approach, Prentice-Hall, Englewood Cliffs.

Doyle, J., Glover, K., Khargonekar, P., Francis, B., 1989, State space solutions to standard $\mathcal{H}^{2}$ and $\mathcal{H}^{\infty}$ control problems, IEEE Transactions on Automatic Control 34, 831-847.

Drazen, A. 2000, Political economy in macroeconomics, (Princeton University Press, Princeton).

Easley, D., Kiefer, N., 1988, Controlling stochastic processes with unknown parameters, Econometrica 56, 1045-1064.

Estrella, A., Mishkin, F. S. 1998, Rethinking the role of NAIRU in monetary policy: implications of model formulation and uncertainty. Unpublished manuscript.

Ferguson, T. 1967, Mathematical statistics, (Academic Press, New York).

Fuhrer, J., Moore, G., 1995a, Inflation persistence, Quarterly Journal of Economics 125, 127159.

Fuhrer, J., Moore, G., 1995b, Monetary policy trade-offs and the correlation between nominal interest rates and real output, American Economic Review 85, 219-239.

Giannoni, M. P., 2000, Does model uncertainty justify caution? Robust optimal monetary policy in a forward-looking model, Macroeconomic Dynamics .

Glover, K., Doyle, J., 1988, State-space formulae for all stabilizing controllers that satisfy an $\mathcal{H}^{\infty}$-norm bound and relations to risk sensitivity, Systems and Control Letters 11, 162172.

Goodfriend, M., 1991, Interest rate smoothing in the conduct of monetary policy, CarnegieRochester Conference Series on Public Policy 34, 7-30.

Hansen, L. P., Sargent, T. J. 1999a, Five games and two objective functions that promote robustness. Unpublished manuscript, U. of Chicago and Hoover Institution.

Hansen, L., Sargent, T. J., 1995, Discounted linear exponential quadratic gaussian control, IEEE Transactions on Automatic Control 40, 968-971.

Hansen, L., Sargent, T. J. 1999b, Robustness and commitment: a monetary policy example. Unpublished manuscript, U. of Chicago and Hoover Institution. 
Hansen, L., Sargent, T. J., Tallarini, T. D., 1999, Robust permanent income and pricing, Review of Economic Studies 66, 873-907.

Johansen, L. 1973, Targets and instruments under uncertainty, in: H. C. Boss, H. Linneman, P. de Wolff eds., Economic structure and development: essays in honor of Jan Tinbergen, (North-Holland, Amsterdam), 3-20.

Johansen, L. 1978, Lectures on macroeconomic planning, (North-Holland, Amsterdam).

Kasa, K., 2000, Model uncertainty, robust policies, and the value of commitment, Macroeconomic Dynamics .

Levin, A., Wieland, V., Williams, J. 1999, Robustness of simple monetary policy rules under model uncertainty, in: J. B. Taylor ed., Monetary policy rules, (University of Chicago Press, Chicago), 263-309.

Macaulay, F. R. 1938, Some theoretical problems suggested by the movements of interest rates, bond yields, and stock prices in the United States since 1956, (National Bureau of Economic Research, Cambridge).

Mercado, R. P., Kendrick, D. A., 1999, Caution in macroeconomic policy: uncertainty and the relative intensity of policy, Economics Letters 68, 37-41.

Newey, W. K., West, K. D., 1987, A Simple positive-definite heteroskedasticity and autocorrelation consistent covariance matrix, Econometrica 55, 703-708.

Onatski, A. 1999, Minimax analysis of model uncertainty: comparison to Bayesian approach, worst possible economies, and optimal robust monetary policies. (Department of Economics, Harvard University).

Onatski, A., Stock, J. H., 2000, Robust monetary policy under model uncertainty in a small model of the U.S. economy, NBER Working Paper Series 7490.

Orphanides, A., 1998, Monetary policy rules based on real time data, Federal Reserve Board Finance and Economics Discussion Series 1998-3.

Orphanides, A., Porter, R. D., Reifschneider, D., Tetlow, R. J., Finan, F. S., 2000, Errors in the measurement of the output gap, and the design of monetary policy, Journal of Economics and Business 52.

Roberts, J. M., 1995, New Keynesian economics and the Phillips curve, Journal of Money Credit and Banking 27, 975-984. 
Rudebusch, G. D. 1998, Is the Fed too timid: monetary policy in an uncertain world? Unpublished manuscript, Federal Reserve Bank of San Francisco.

Rudebusch, G. D., Svensson, L. E. O. 1999, Policy rules for inflation targeting, in: J. B. Taylor ed., Monetary policy rules, (University of Chicago Press, Chicago), 203-262.

Sack, B., 1998a, Does the fed act gradually? A var analysis, Federal Reserve Board Finance and Economics Discussion Series 1998-17.

Sack, B., 1998b, Uncertainty, learning, and gradual monetary policy, Federal Reserve Board Finance and Economics Discussion Series 1998-34.

Sack, B., Wieland, V., 2000, Interest-rate smoothing and optimal monetary policy, Journal of Economics and Business 52.

Sargent, T. J. 1999, Discussion of 'policy rules for open economies' by Laurence Ball, in: J. B. Taylor ed., Monetary policy rules, (University of Chicago Press, Chicago), 144-54.

Smets, F. 1999, Output gap uncertainty: does it matter for the Taylor rule?, in: B. Hunt, A. Orr eds., Monetary policy under uncertainty, (Reserve Bank of New Zealand, Wellington), $10-29$.

Söderström, U., 1999a, Should central banks be more aggressive?, Stockholm University, Working Papers in Economics and Finance 309.

Söderström, U., 1999b, Monetary policy with uncertain parameters, Stockholm University, Working Papers in Economics and Finance 308.

Sworder, D. D., 1964, Minmax control of discrete time stochastic systems, Society for Industrial Aplied Mathematics, Series A, Control 2 433-449.

Taylor, J. B., 1980, Aggregate dynamics and staggered contracts, Journal of Political Economy $88,1-23$.

Taylor, J. B., 1993, Discretion versus policy rules in practice, Carnegie-Rochester Conference Series on Public Policy 39, 195-214.

Tetlow, R. J. 2000, Uncertain potential output and monetary policy in a forward-looking model. Unpublished manuscript, Board of Governors of the Federal Reserve System.

Whittle, P. 1990, Risk-sensitive optimal control, Wiley, New York. 
Wieland, V., 1998, Monetary policy and uncertainty about the natural unemployment rate, Federal Reserve Board Finance and Economics Discussion Series 1998-2.

Woodford, M., 1999, Optimal monetary policy inertia, Manchester School 67, 1-35.

Zames, G., 1981, Feedback and optimal sensitivity: model reference transformations, multiplicative seminorms, and approximate inverses, IEEE Transactions on Automatic Control AC-26, 301-320.

Zhou, K., Doyle, J. C., Glover, K. 1996, Robust and optimal control, (Prentice-Hall, Englewood Cliffs). 
Figure 1: Frequency Decomposition of Expected Losses under Model and Shock Uncertainty

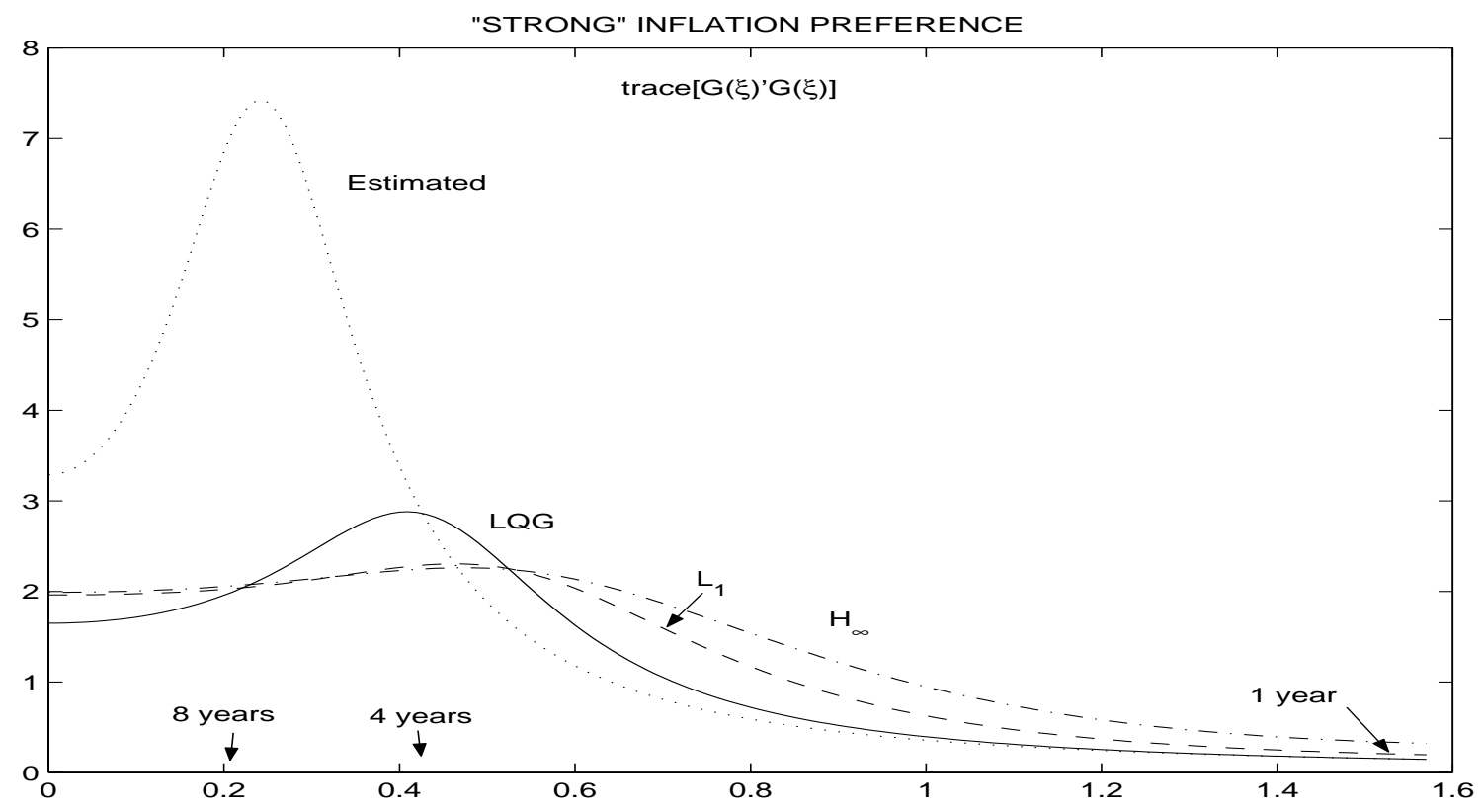

Figure 2: Frequency Decomposition of Expected Losses under Model and Shock Uncertainty

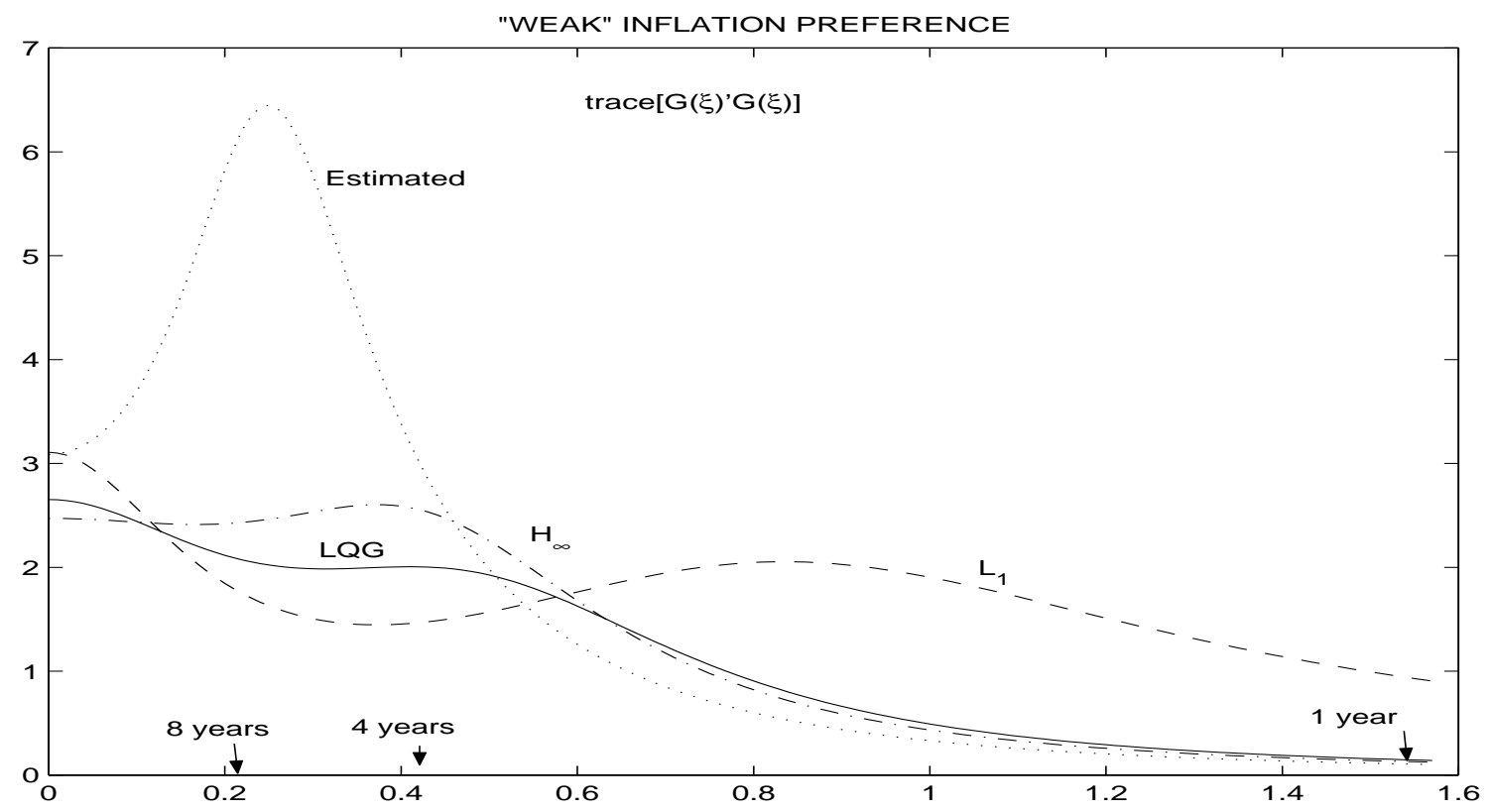


Figure 3: Frequency Decomposition of Expected Losses: Perturbations to all Parameters

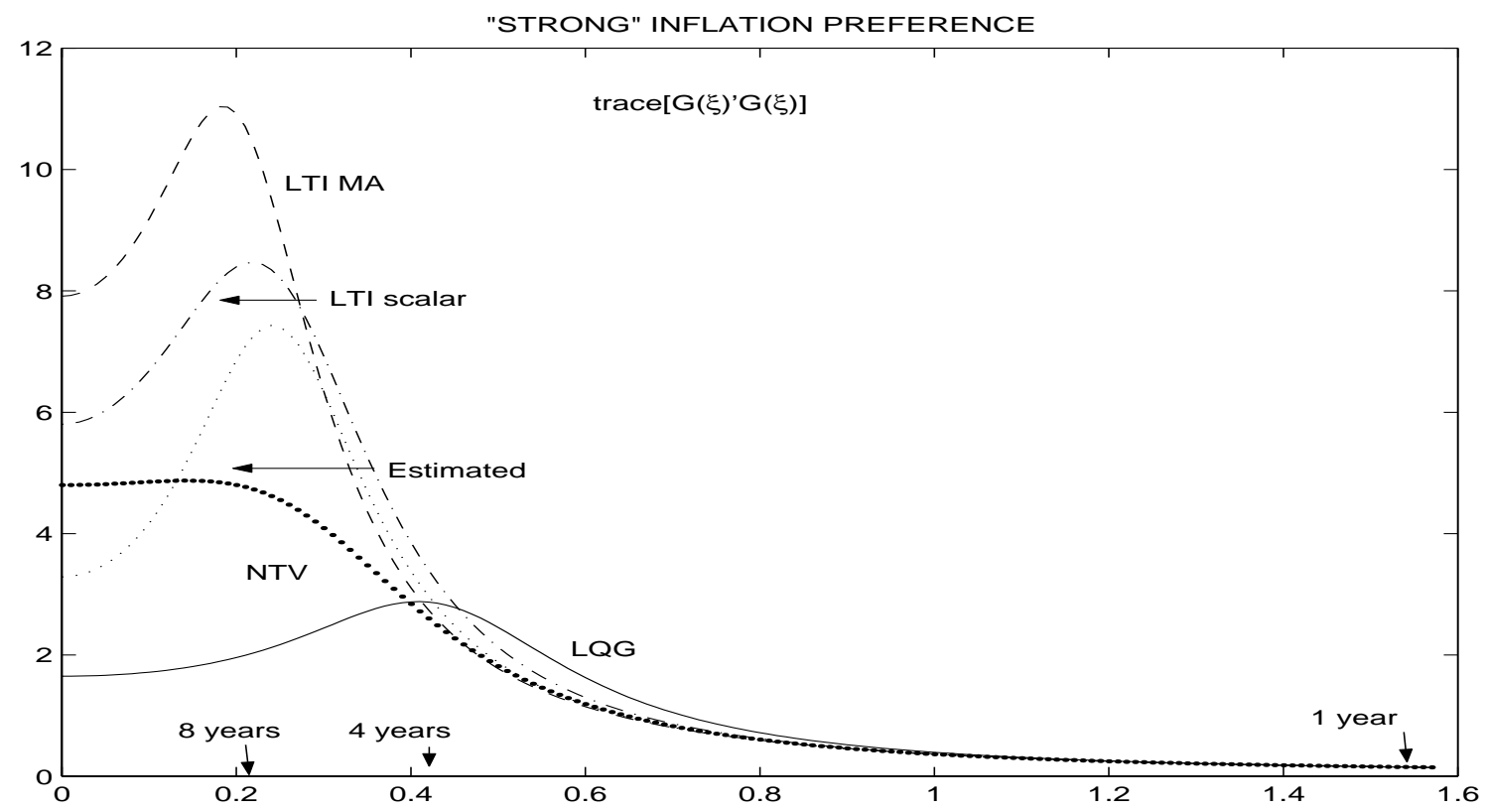

Figure 4: Frequency Decomposition of Expected Losses: Perturbations to all Parameters

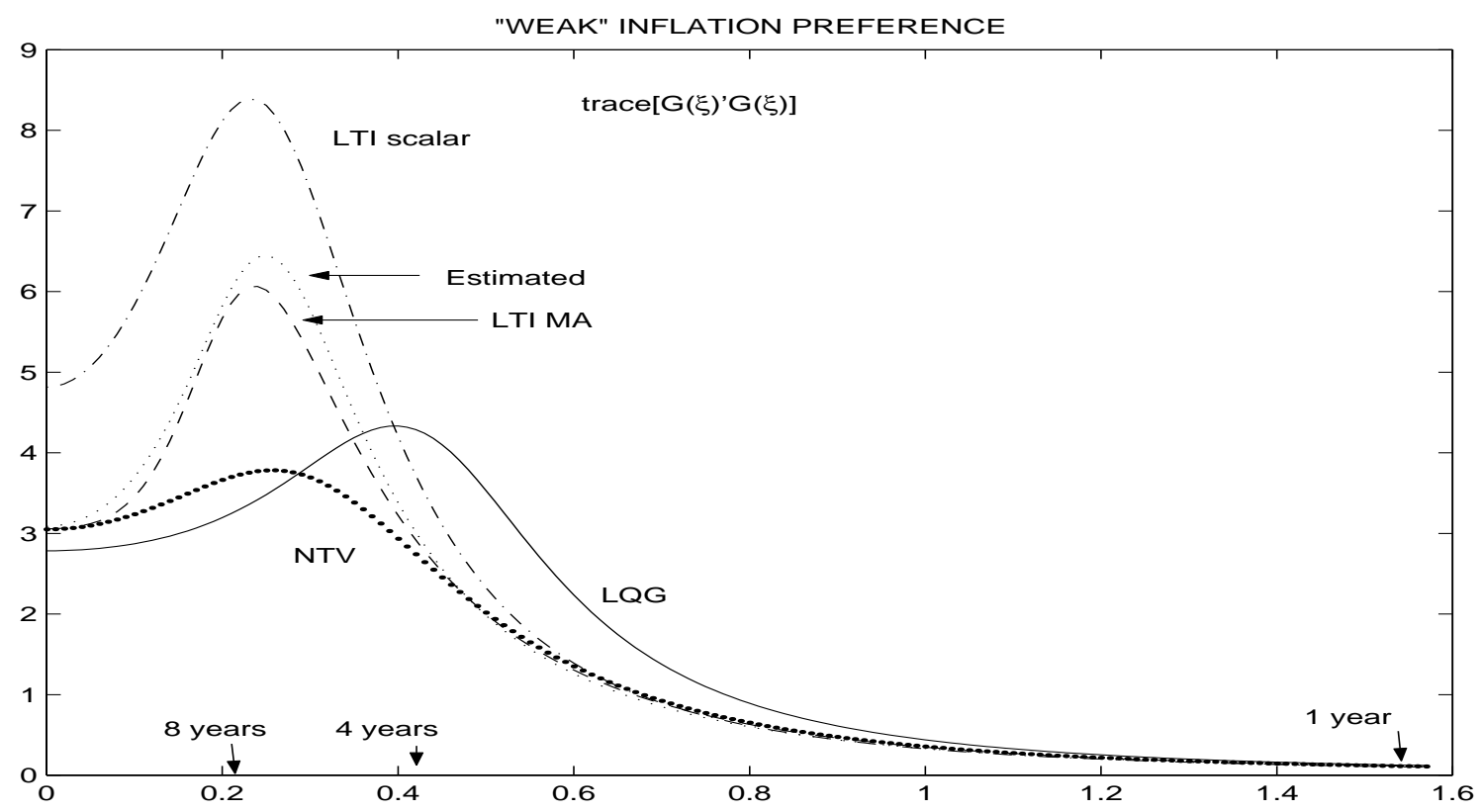


Figure 5: Frequency Decomposition of Expected Losses: Perturbations to Lagged Output Coefficients

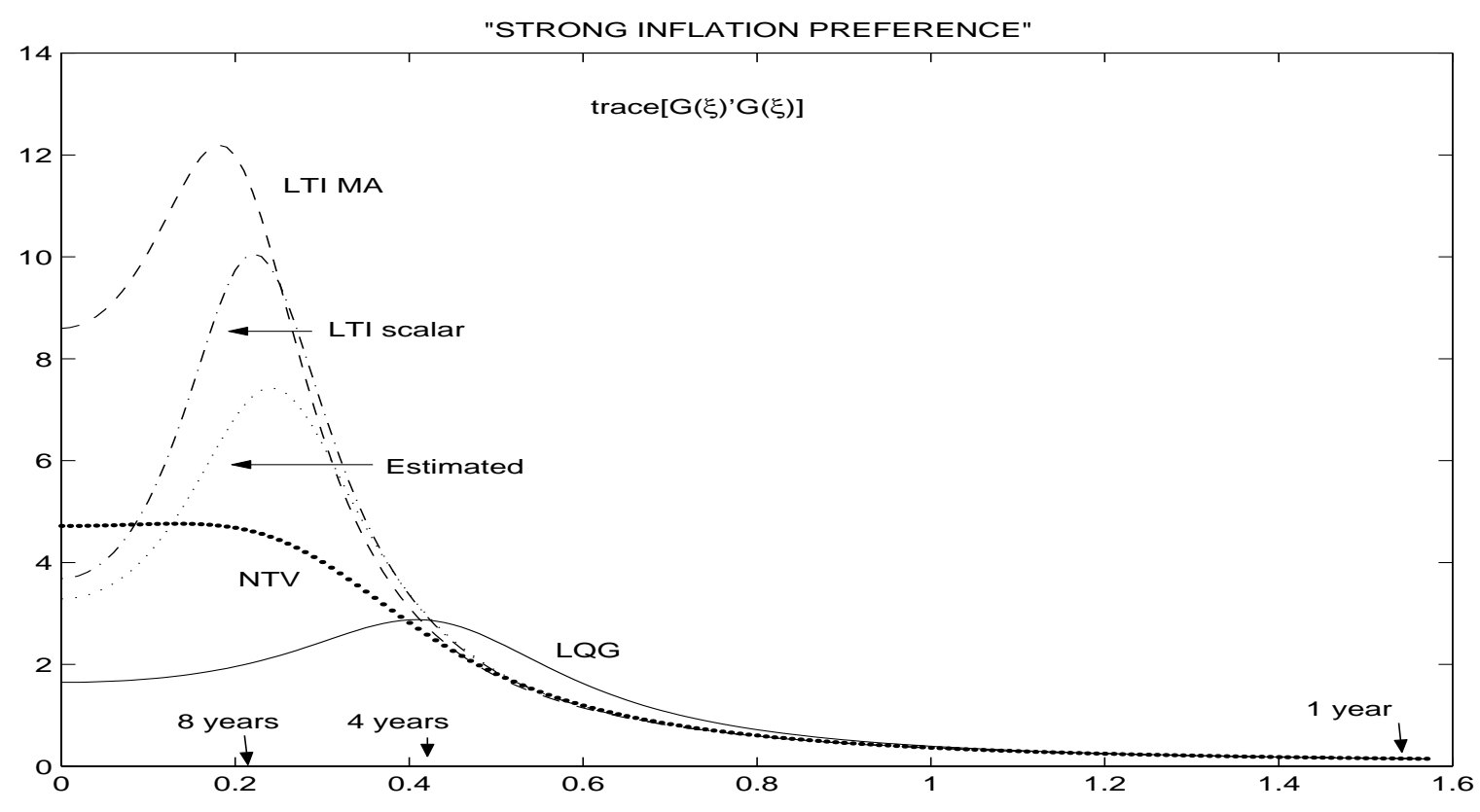

Figure 6: Frequency Decomposition of Expected Losses: Perturbations to Lagged Output Coefficients

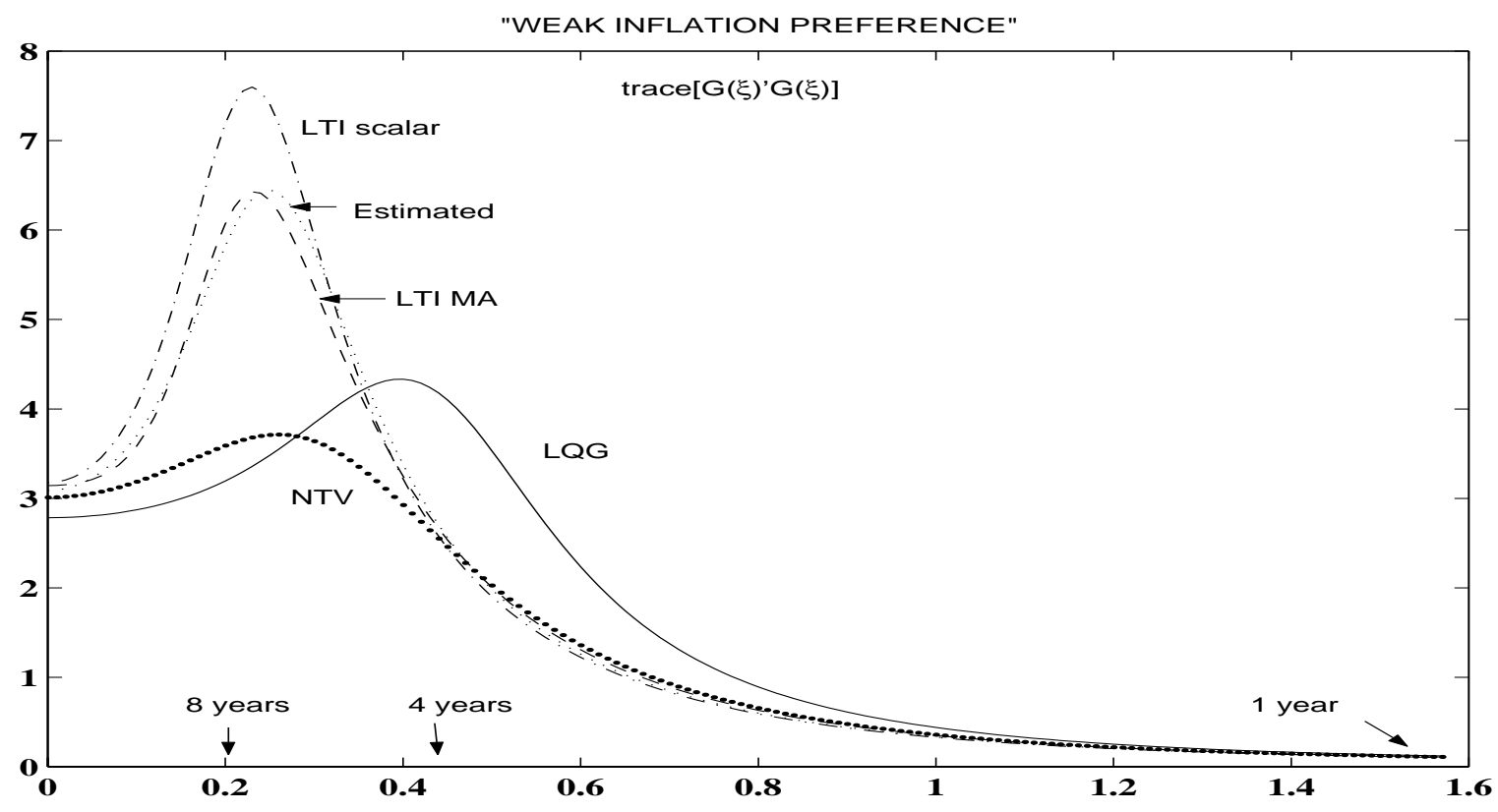


Figure 7: Frequency Decomposition of Expected Losses: Perturbations to Slope of IS Curve

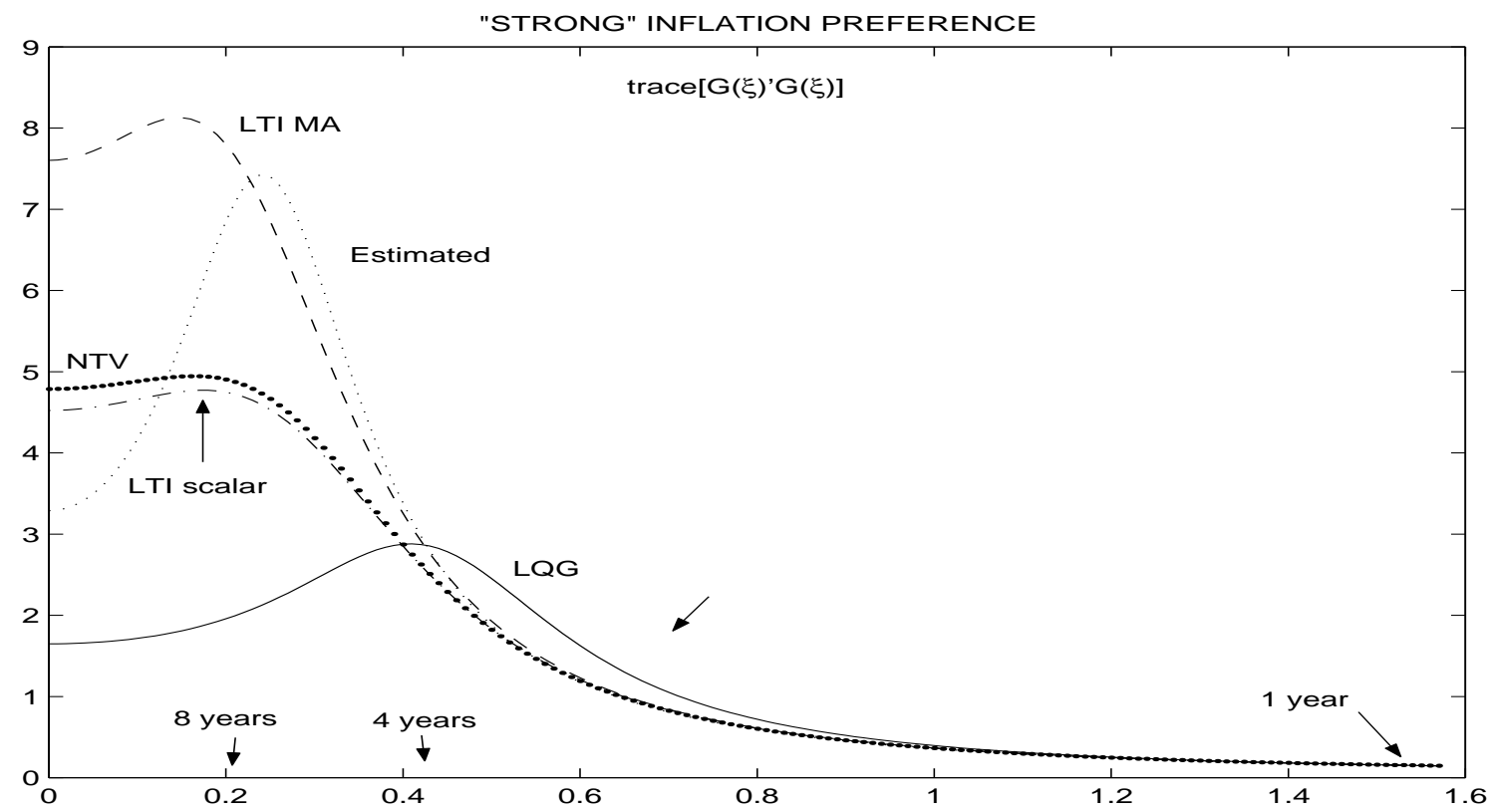

Figure 8: Frequency Decomposition of Expected Losses: Perturbations to Slope of IS Curve

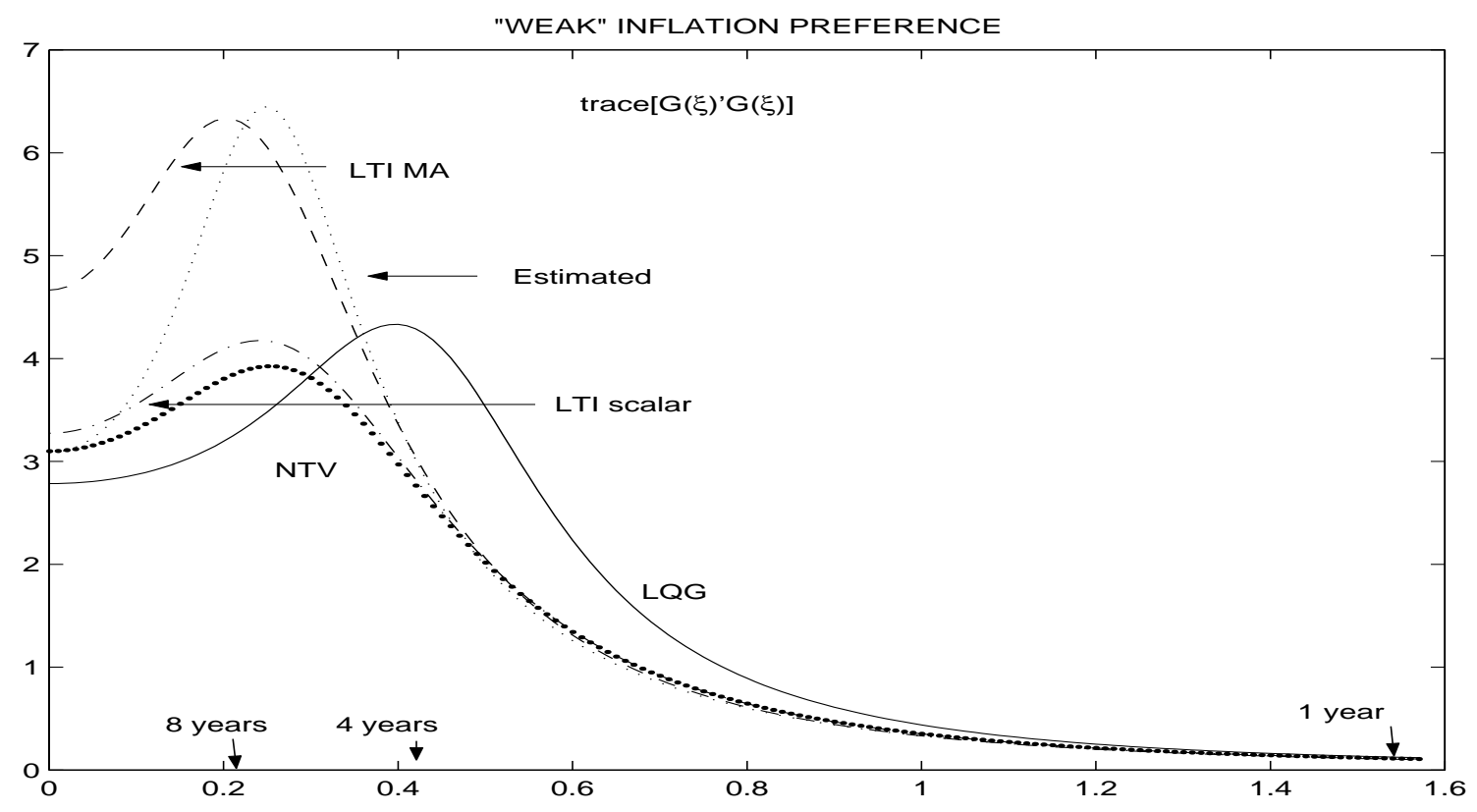


Figure 9: Frequency Decomposition of Expected Losses: Perturbations to Slope of Phillips Curve

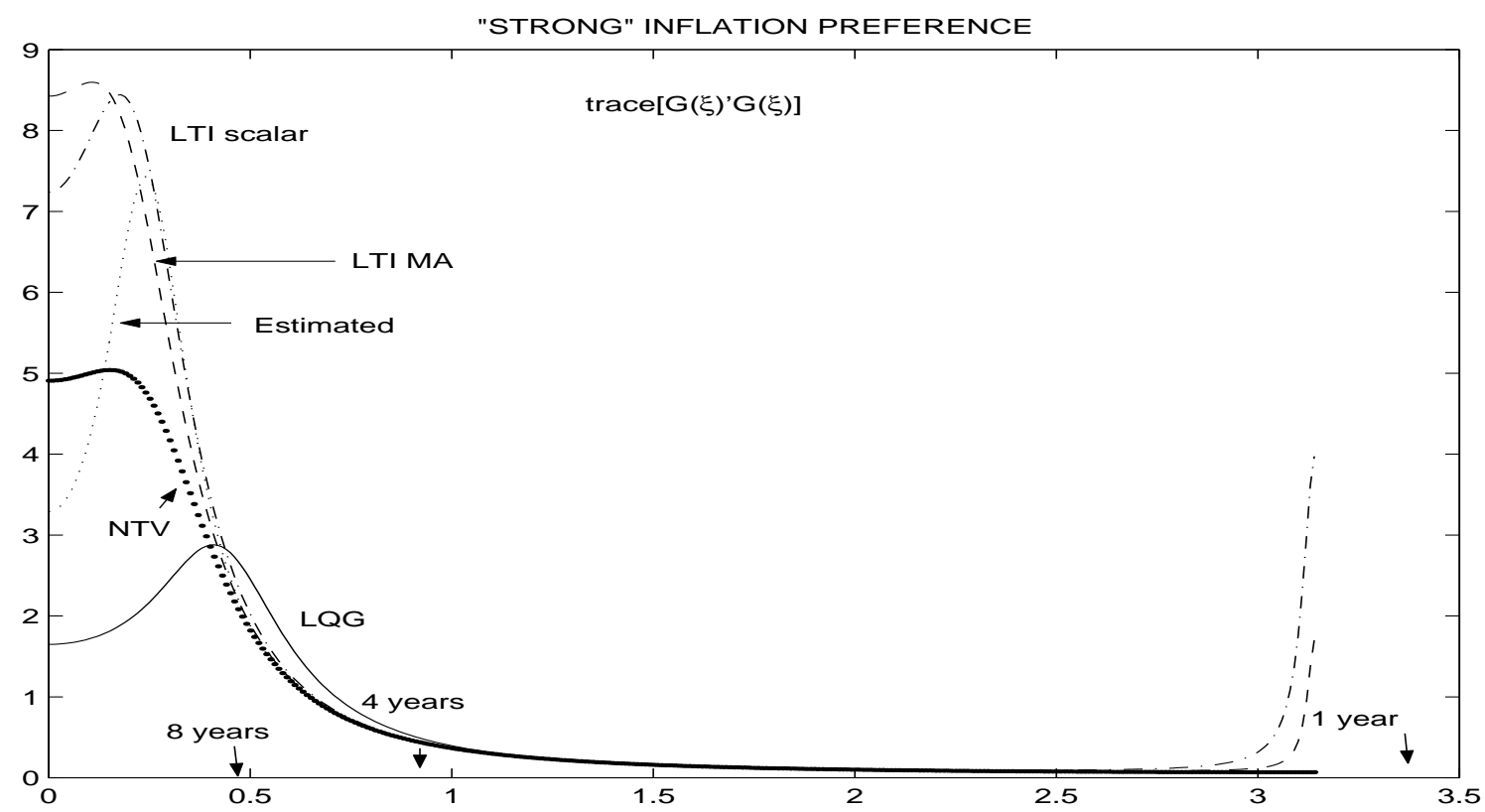

Figure 10: Frequency Decomposition of Expected Losses: Perturbations to Slope of Phillips Curve

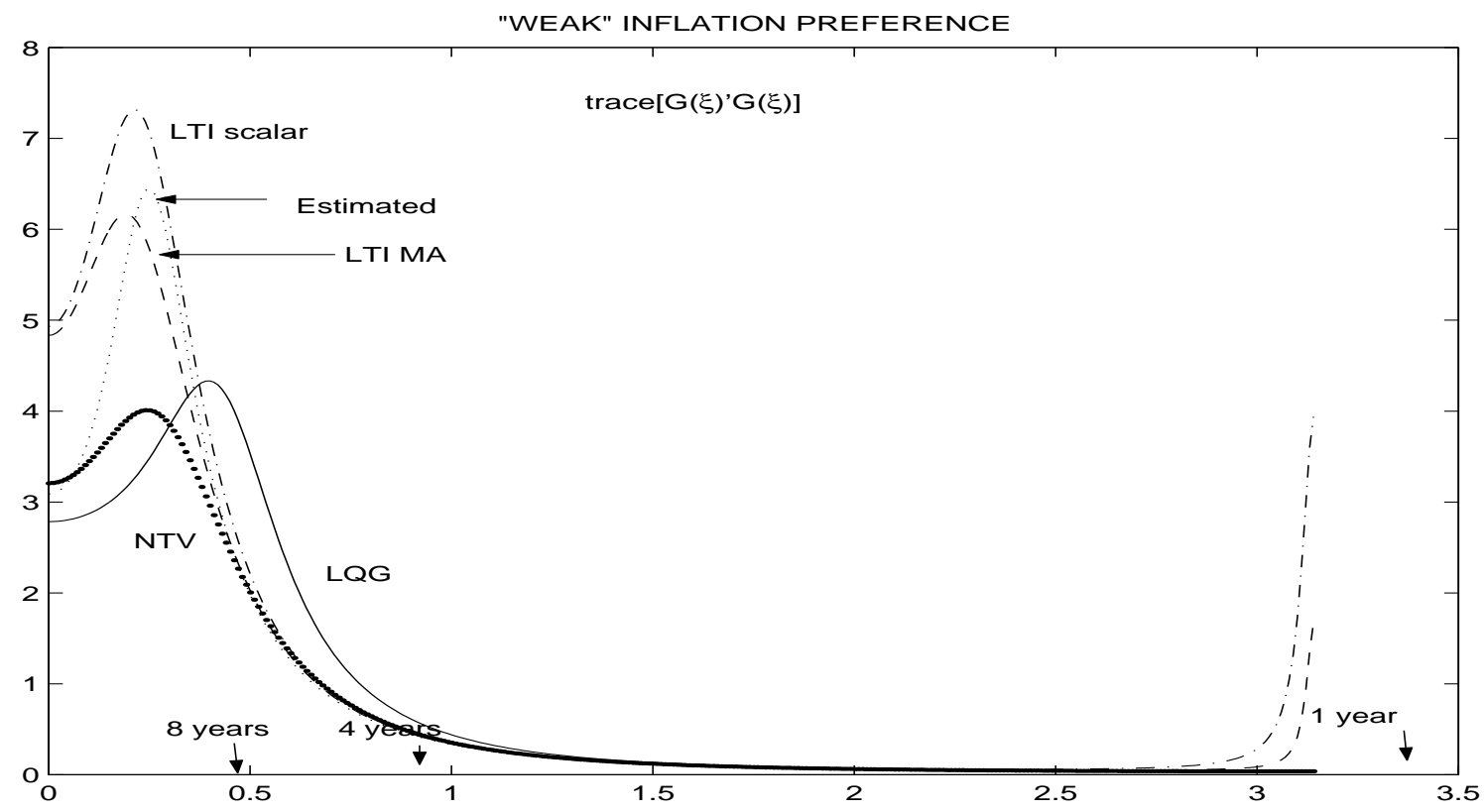

